\title{
Canonical relativistic quantization of electromagnetic field in the presence of an anisotropic conductor magneto-dielectric medium
}

\author{
Majid Amooshahi \\ Department of Physics, Faculty of Science, \\ University of Isfahan, Isfahan, Iran \\ amooshahi@sci.ui.ac.ir
}

Received 1 September 2017

Accepted 26 November 2017

Published 11 December 2017

\begin{abstract}
A canonical relativistic quantization of the electromagnetic field is introduced in the presence of an anisotropic conductor magneto-dielectric medium in a standard way in the Gupta-Bleuler framework. The medium is modeled by a continuum collection of the vector fields and a continuum collection of the antisymmetric tensor fields of the second rank in Minkowski space-time. The collection of vector fields describes the conductivity property of the medium and the collection of antisymmetric tensor fields describes the polarization and the magnetization properties of the medium. The conservation law of the total electric charges, induced in the anisotropic conductor magneto-dielectric medium, is deduced using the antisymmetry conditions imposed on the coupling tensors that couple the electromagnetic field to the medium. Two relativistic covariant constitutive relations for the anisotropic conductor magneto-dielectric medium are obtained. The constitutive relations relate the antisymmetric electric-magnetic polarization tensor field of the medium and the free electric current density four-vector, induced in the medium, to the strength tensor of the electromagnetic field, separately. It is shown that for a homogeneous anisotropic medium the susceptibility tensor of the medium satisfies the Kramers-Kronig relations. Also it is shown that for a homogeneous anisotropic medium the real and imaginary parts of the conductivity tensor of the medium satisfy the Kramers-Kronig relations and a relation other than the Kramers-Kronig relations.

Keywords: Anisotropic conductor magneto-dielectric medium; electric-magnetic polarization tensor; free electric current density four-vector; relativistic covariant constitutive relations; susceptibility tensor; conductivity tensor; Kramers-Kronig relations; Minkowski space-time; relativistic quantum electrodynamics.
\end{abstract}

PACS numbers: $12.20 . \mathrm{Ds}, 42.50 . \mathrm{Nn}$

This is an Open Access article published by World Scientific Publishing Company. It is distributed under the terms of the Creative Commons Attribution 4.0 (CC-BY) License. Further distribution of this work is permitted, provided the original work is properly cited. 


\section{Introduction}

There is a quantization method of the electromagnetic field in the presence of dispersive and absorbing dielectric media known as the Green function method. ${ }^{1-7}$ In this quantization scheme the causal constitutive relation of the dielectric medium is written in frequency domain. Then, the noise polarization density is added, phenomenologically, to the constitutive relation of the dielectric medium. Combination of the constitutive relation of the dielectric medium and the Maxwell's equations, in the frequency domain, gives the wave equation for the temporal Fourier transform of the electric field. The noise polarization density is appeared on the right-hand side of the wave equation as a source term. The electric field, in frequency domain, is written in terms of the dyadic Green function of the wave equation and the noise polarization density. The shape of the dyadic Green function of the wave equation is dependent on the configuration of the dispersive dielectric medium and should satisfy the suitable boundary conditions on the surfaces of discontinuity of the dielectric medium. The boundary conditions for the dyadic Green function are deduced from the boundary conditions of the electromagnetic field on the surfaces of discontinuity of the dielectric medium. The expression of the electric field in terms of the dyadic Green function and the added noise polarization density in frequency domain is, in fact, as a mode expansion for the electric field. The noise polarization density is related to some bosonic operators in frequency domain. The suitable commutation relations are imposed on the bosonic operators, so that the electromagnetic field satisfies the same equal-time commutation relations as in free space. ${ }^{5}$ This quantization method of electromagnetic field has been generalized in the presence of magneto-dielectric media,,$-\frac{8}{-10}$ in the presence of amplifying media ${ }^{11,12}$ and in the presence of external point charge particles. ${ }^{10}$ We can refer to some applications of this quantization scheme, for example, the calculation of the decay rate of an initially excited two-level atom in the presence of absorbing media,,$\underline{13}-24$ the calculation of the Casimir forces on the dispersive and absorbing media ${ }^{9,25-}-51$ and the Cherenkov radiation of a point charge particle in the presence of absorbing media. $\frac{52,53}{}$

There is a second fully canonical nonrelativistic quantization approach of the electromagnetic field in the presence of absorbing anisotropic magneto-dielectric media with spatial-temporal dispersion. ${ }^{54}$ In this quantization method the magnetodielectric medium is modeled by two independent continuum collections of spacetime dependent three-dimensional harmonic oscillators. One of the collections describes the electric property of the medium and the other collection describes the magnetic property of the medium. Two continuum sets of the coupling tensors of the second rank, in usual three-dimensional space, are applied in the interaction part of the Lagrangian of the total system (the electromagnetic field plus medium). The coupling tensors couple the electromagnetic field to the anisotropic magneto-dielectric medium. The coupling tensors play a key role in this canonical quantization scheme, so that the electric and magnetic polarization densities of 
the medium are defined in terms of the coupling tensors and the harmonic oscillator fields modeling the medium. Also the electric and the magnetic susceptibility tensors of the magneto-dielectric medium are expressed in terms of the coupling tensors. It is shown that the susceptibility tensors of the anisotropic magneto-dielectric medium satisfy the Kramers-Kronig relations as this should be so for any memory function of a causal relation. $\frac{55}{}$ A canonical quantization is achieved for both the electromagnetic field and the absorbing anisotropic magneto-dielectric medium. The constitutive relations of the anisotropic magneto-dielectric medium are extracted in the Heisenberg picture. The noise electric and magnetic polarization densities of the magneto-dielectric medium are obtained in terms of the coupling tensors that couple the electromagnetic field to the medium and the harmonic oscillators fields modeling the medium at $t=0$.

This nonrelativistic canonical quantization scheme of the electromagnetic field has been generalized in presence of absorbing bi-anisotropic magneto-dielectric media. ${ }^{56}$ In this case, four continuum sets of the coupling tensors of the second rank, in usual three-dimensional space, are applied in the interaction part of the Lagrangian of the total system. The coupling tensors couple the electromagnetic field to the bi-anisotropic magneto-dielectric medium. The electric and magnetic polarization densities of the medium are defined in terms of the space-time dependent harmonic oscillators fields modeling the medium and the coupling tensors that couple the electromagnetic field to the medium. The constitutive relations of the medium relating the electric polarization and the magnetic polarization densities of the bi-anisotropic magneto-dielectric medium, separately, to both the electric and the magnetic fields are obtained in the Heisenberg picture. The four susceptibility tensors of the bi-anisotropic magneto-dielectric medium, appeared in the constitutive relations of the medium, are obtained in terms of the coupling tensors that couple the electromagnetic field to the medium. It is shown that the four susceptibility tensors of the bi-anisotropic magneto-dielectric medium satisfy the Kramers-Kronig relations. $\frac{55}{5}$

This quantization method of the electromagnetic field has been applied in the presence of a nonlinear anisotropic magneto-dielectric medium with spatialtemporal dispersion. ${ }^{57}$ In this case, the interaction part of the Lagrangian of the total system is written as a series of different powers of the space-time dependent three-dimensional harmonic oscillators modeling the nonlinear anisotropic magneto-dielectric medium. Some coupling tensors of various ranks, in the usual three-dimensional space, are applied in the interaction part of the Lagrangian of the total system. The coupling tensors couple the electromagnetic field to the nonlinear anisotropic magneto-dielectric medium. The electric and magnetic polarization densities of the medium are expressed as a series of the different powers of the harmonic oscillator fields, modeling the medium, and the coupling tensors that couple the electromagnetic field to the medium. A canonical quantization of both the electromagnetic field and the nonlinear anisotropic magneto-dielectric medium is 


\section{Amooshahi}

provided. The constitutive relations of the medium are obtained in the Heisenberg picture. The constitutive relations of the medium relate the electric and magnetic polarization densities of the anisotropic nonlinear magneto-dielectric medium to the electric and magnetic fields, respectively, as a series of different powers of the electric and magnetic fields. The susceptibility tensors of the medium of the various ranks, appeared in the constitutive relations of the medium, are obtained in terms of the coupling tensors that couple the electromagnetic to the nonlinear anisotropic magneto-dielectric medium. Imposing some symmetry relations on the coupling tensors of the electromagnetic field and the medium caused the susceptibility tensors of the medium of various ranks obey some symmetry relations which may be found in the literatures in nonlinear optics. $\frac{58}{}$

This quantization method can be generalized to the case of moving magnetodielectric media in a complete canonical covariant relativistic language. $\stackrel{59}{5}$

In this paper, a fully canonical relativistic quantization of the electromagnetic field is introduced in the presence of an anisotropic conductor polarizable and magnetizable medium in three steps.

In the first step an anisotropic magneto-dielectric (but not conductor) medium is modeled by a continuum collection of antisymmetric tensor fields of the second rank in Minkowski space-time. A Lagrangian density is proposed for the electromagnetic field together the anisotropic magneto-dielectric medium which is a scalar under Poincaré transformation. A continuum set of the coupling tensor fields of the fourth rank, in Minkowski space-time, is applied in the interaction part of the Lagrangian density of the total system (the electromagnetic field plus the magneto-dielectric medium). The coupling tensors couple the electromagnetic field to the anisotropic magneto-dielectric medium. The antisymmetric electric-magnetic polarization tensor of the medium is defined in terms of the antisymmetric tensor fields modeling the magneto-dielectric medium and the coupling tensors that couple the electromagnetic field to the medium. The relativistic covariant constitutive relation of the anisotropic magneto-dielectric medium is obtained relating the antisymmetric electric-magnetic polarization tensor of the magneto-dielectric medium to the strength tensor of the electromagnetic field. The susceptibility tensor of the anisotropic magneto-dielectric medium is expressed in terms of the retarded Green function and the coupling tensor fields that couple the electromagnetic field to the medium. It is shown that for a homogeneous anisotropic bulk magneto-dielectric medium the susceptibility tensor satisfy the Kramers-Kronig relations in frequency domain. A canonical relativistic quantization of the both the electromagnetic field and the magneto-dielectric medium is provided in the Gupta-Bleuler formalism. 60 The noise antisymmetric electric-magnetic polarization tensor of the anisotropic magneto-dielectric medium is expanded in terms of the ladder operators of the total system (the electromagnetic field plus the medium) and the coupling tensors that couple the electromagnetic field to the medium. 
In the second step an anisotropic conductor (but not polarizable and magnetizable) medium is modeled by a continuum collection of vector fields in Minkowski space-time. A Lagrangian density is provided for the total system (the electromagnetic field plus the conductor medium) that is invariant under Poincaré transformation. A continuum set of the coupling tensors of the fourth rank in Minkowski space-time is introduced in the interaction part of the Lagrangian of the total system. The coupling tensors couple the electromagnetic field to the anisotropic conductor medium. Some antisymmetry conditions are imposed on the coupling tensors of the electromagnetic field and the conductor medium. The free electric current density four-vector induced in the conductor medium is defined in terms of the vector fields modeling the medium and the coupling tensor fields that couple the electromagnetic field to the medium. The conservation law of the free electric charges induced in the conductor medium is extracted from antisymmetry conditions imposed on the coupling tensors that couple the electromagnetic field to the conductor medium. The relativistic covariant constitutive relation of the anisotropic conductor medium is obtained relating the free electric current density four-vector induced in the medium to the strength tensor of the electromagnetic field. The conductivity tensor of the anisotropic conductor medium is defined in terms of the spatial-temporal derivatives of the retarded Green function and the coupling tensor fields that couple the electromagnetic field to the medium. It is shown that the real and imaginary parts of the conductivity tensor of an anisotropic bulk conductor medium, in frequency domain, are related to each other through the Kramers-Kronig relations and a relation other than the Kramers-Kronig relation. A canonical relativistic quantization of the total system (the electromagnetic field and the conductor medium) is achieved in a standard way in the Gupta-Bleuler method. 60 The noise free electric current density four-vector induced in the conductor medium is expressed in terms of the ladder operators of the total system (the electromagnetic field plus the conductor medium) and the coupling tensors that couple the electromagnetic field to the conductor medium.

In the last step an anisotropic conductor polarizable and magnetizable medium is modeled by a continuum collection of the vector fields and a continuum collection of the antisymmetric tensor fields in Minkowski space-time. The vector fields describe the conductivity property of the medium and the antisymmetric tensor fields describe the polarization and the magnetization properties of the medium. A Lagrangian density is proposed for the total system (the electromagnetic field plus the anisotropic conductor magneto-dielectric medium) that is a scalar under Poincaré transformation. The electromagnetic field is coupled to the vector fields describing the conductivity property of the medium by a continuum set of the coupling tensor fields of the fourth rank in Minkowski space-time. Also the electromagnetic field is coupled to the antisymmetric tensor fields describing the polarization and the magnetization properties of the medium by another tensor fields set of the fourth rank in Minkowski space-time. The free electric current density 


\section{Amooshahi}

four-vector induced in the medium is defined in terms of the vector fields describing the conductivity property of the medium and the coupling tensors that couple the electromagnetic field to these vector fields. The antisymmetric electric-magnetic polarization tensor of the medium is expressed in terms of the antisymmetric tensor fields describing the polarization and the magnetization properties of the medium and the coupling tensors that couple the electromagnetic field to these antisymmetric tensor fields. The conservation law of the total electric charges induced in the anisotropic conductor magneto-dielectric medium is deduced using the antisymmetry conditions imposed on the coupling tensors that couple the electromagnetic field to the medium. Two relativistic covariant constitutive relations for the anisotropic conductor magneto-dielectric medium are obtained. One of the constitutive relations of the medium relates the free electric current density four-vector induced in the medium to the strength tensor of the electromagnetic field. Another constitutive relation of the medium relates the antisymmetric electric-magnetic polarization tensor field of the medium to the strength tensor of the electromagnetic field. The susceptibility tensor of the medium is defined in terms of the retarded Green function and the coupling tensors that couple the electromagnetic field to the antisymmetric tensor fields describing the polarization and the magnetization properties of the medium. The conductivity tensor of the medium is expressed in terms of the spatial-temporal derivatives of the retarded Green function and the coupling tensors that couple the electromagnetic field to the vector fields describing the conductivity property of the medium. The susceptibility and the conductivity tensors of the medium have the same physical and mathematical features of the previous two steps mentioned above. The electromagnetic field together the anisotropic conductor magneto-dielectric medium is quantized in a canonical relativistic standard way using the Gupta-Bleuler method. ${ }^{60}$ The total noise electric current density four-vector induced in anisotropic conductor magneto-dielectric medium is written in terms of the ladder operators of the total system and the coupling tensors that couple the electromagnetic field to the medium. The quantum relativistic covariant wave equation of the four-vector potential of the electromagnetic field is obtained in the presence of the anisotropic conductor polarizable and magnetizable medium. The quantum relativistic covariant wave equation of the four-vector potential is solved for a homogeneous anisotropic conductor magneto-dielectric bulk medium using the four-dimensional Fourier transform technique.

Throughout this paper the canonical relativistic quantization of the electromagnetic field is achieved in the Gupta-Bleuler framework. ${ }^{60}$ This means that the Lorentz gauge $\partial_{\mu} A^{\mu}(x)=0$ is not applied directly on the four-vector potential operator $A^{\mu}(x)$ of the electromagnetic field. In the Gupta-Bleuler formalism, those states $|\psi\rangle$ in the Fock space of the total system (the electromagnetic field plus the medium) for calculations of the physical quantities are admissible that the expectation value of the phrase $\partial_{\mu} A^{\mu}(x)$ is zero, that is $\left\langle\psi\left|\partial_{\mu} A^{\mu}(x)\right| \psi\right\rangle=0$. Also throughout this paper $x=\left(x_{0}=c t, \mathbf{x}\right)$ denotes the space-time points and the space-time metrics is taken as $g=\operatorname{diag}(1,-1,-1,-1)$. 


\section{Anisotropic Polarizable and Magnetizable Medium}

\subsection{Relativistic classical Euler-Lagrange equations}

In order to canonical quantization of the electromagnetic field in the presence of an anisotropic polarizable and magnetizable (but not conductor) medium in a relativistic covariant language, we model the medium by a continuum set of the antisymmetric tensor fields $Y^{\alpha \beta}(\omega, x)$ of the second rank in Minkowski space-time labeled by a real positive continuous parameter $\omega$. The tensor fields $Y^{\alpha \beta}(\omega, x)$ describe the electric together the magnetic properties of the polarizable and magnetizable medium. So that, as we will see the antisymmetric electric-magnetic polarization tensor field of the medium is defined in terms of the tensor fields $Y^{\alpha \beta}(\omega, x)$ and the coupling tensors that couple the electromagnetic field to the medium. The choice of the continuum set $Y^{\alpha \beta}(\omega, x), \omega \in(0, \infty)$ to model the polarizable and magnetizable medium, in order to quantize the electromagnetic field in a relativistic covariant formulation, is in correspondence to the canonical quantization of the electromagnetic field in the presence of a magneto-dielectric medium in a nonrelativistic quantization scheme in viewpoint of mathematical structure. In the nonrelativistic canonical quantization scheme of the electromagnetic field in the presence of a magneto-dielectric medium ${ }^{54,56}$ the medium is modeled by two independent continuum collections of the space-time dependent three-dimensional harmonic oscillators each labeled by a real positive continuous parameter $\omega$. One of the collection of the harmonic oscillator fields describes the electric property and the second collection describes the magnetic property of the medium. So that, for any real positive parameter $\omega$ there is six independent components for the dynamical variables describing the polarization and the magnetization properties of the medium. In the present relativistic quantization scheme of the electromagnetic field in the presence of a magneto-dielectric medium, the antisymmetric tensor fields $Y^{\alpha \beta}(\omega, x)$, describing both the electric and the magnetic properties of the medium, also have six independent components in Minkowski space-time for any real positive parameter $\omega$.

In order to have a canonical relativistic quantization formulation for both the electromagnetic field and the magneto-dielectric medium, we need a Lagrangian density which is a scalar under Poincaré transformation. The Lagrangian density of the total system (the electromagnetic field plus the medium) is proposed as

$$
\begin{aligned}
£(x)= & -\frac{1}{2} \partial_{\mu} A_{\nu}(x) \partial^{\mu} A^{\nu}(x)-\int_{0}^{\infty} d \omega f^{\mu \nu \alpha \beta}(\omega, x) \partial_{\mu} A_{\nu}(x) Y_{\alpha \beta}(\omega, x) \\
& +\frac{1}{2} \int_{0}^{\infty} d \omega\left[\partial_{\mu} Y_{\alpha \beta}(\omega, x) \partial^{\mu} Y^{\alpha \beta}(\omega, x)-\omega^{2} Y_{\alpha \beta}(\omega, x) Y^{\alpha \beta}(\omega, x)\right],
\end{aligned}
$$

where $x=\left(x_{0}=c t, x^{1}, x^{2}, x^{3}\right)$ is a point in the four-dimensional Minkowski spacetime, $A^{\nu}, \nu=0,1,2,3$ is the four-vector potential of the electromagnetic field. In the Lagrangian density (1) the tensors $f^{\mu \nu \alpha \beta}(\omega, x), \omega \in(0, \infty)$ are the coupling 
tensors of the electromagnetic field and the magneto-dielectric medium and we impose the antisymmetric relations

$$
f^{\mu \nu \alpha \beta}(\omega, x)=-f^{\nu \mu \alpha \beta}(\omega, x), \quad f^{\mu \nu \alpha \beta}(\omega, x)=-f^{\mu \nu \beta \alpha}(\omega, x)
$$

on them. The imposition of the antisymmetry relations (2) on the coupling tensors $f^{\mu \nu \alpha \beta}(\omega, x)$ are in correspondence to the nonrelativistic canonical quantization scheme of the electromagnetic field in the presence of bi-anisotropic magnetodielectric media ${ }^{56}$ in viewpoint of the mathematical structure. In the present relativistic quantization of the electromagnetic field in the presence of a magnetodielectric medium, we will see that the magneto-dielectric medium becomes a bianisotropic one. That is the electric and magnetic polarization densities of the medium, separately, are related to both the electric and magnetic fields. In the nonrelativistic canonical quantization of the electromagnetic field in the presence of a bi-anisotropic magneto-dielectric medium ${ }^{56}$ there are four continuum sets of the coupling tensors of the second rank, in usual three-dimensional space, which couple the electromagnetic field to the medium. The four coupling tensors have totally 36 independent components, in usual three-dimensional space, for each real positive parameter $\omega$. In the present relativistic quantization method, according to the antisymmetry relations (2), the coupling tensors $f^{\mu \nu \alpha \beta}(\omega, x)$, applied in the Lagrangian density (1), also possess precisely 36 independent components in Minkowski space-time for any positive parameter $\omega$. Furthermore, in the present relativistic quantization scheme, we will see that the susceptibility tensor of the magneto-dielectric medium, appeared in relativistic covariant constitutive relation of the medium, is a tensor of the fourth rank in Minkowski space-time. The antisymmetry relations (2) are caused the susceptibility tensor of the medium to be antisymmetric with respect to the first pair and the last pair of its indices. Such a tensor has 36 independent components in Minkowski space-time. In the nonrelativistic electrodynamics the electric and magnetic polarization densities of a bi-anisotropic magneto-dielectric medium, separately, are related to both the electric and magnetic fields by four susceptibility tensors of the second rank in usual three-dimensional space which also have totally 36 independent components.

Using the Lagrangian density (1), the classical relativistic covariant EulerLagrange equation of the four-vector potential of the electromagnetic field is obtained as

$$
\frac{d}{d x^{\mu}}\left(\frac{\partial £}{\partial\left(\partial_{\mu} A_{\nu}(x)\right)}\right)-\frac{\partial £}{\partial\left(A_{\nu}(x)\right)}=0 \Rightarrow-\partial_{\mu} \partial^{\mu} A^{\nu}(x)=4 \pi \partial_{\mu} K^{\mu \nu}(x)
$$

where $K^{\mu \nu}(x)$ is the antisymmetric electric-magnetic polarization tensor of the magneto-dielectric medium and defined in terms of the antisymmetric tensor fields $Y^{\alpha \beta}(\omega, x)$, modeling the magneto-dielectric medium and the coupling tensor 
fields $f^{\mu \nu \alpha \beta}(\omega, x)$ as

$$
\begin{aligned}
K^{\mu \nu}(x)= & \frac{1}{4 \pi} \int_{0}^{\infty} d \omega f^{\mu \nu \alpha \beta}(\omega, x) Y_{\alpha \beta}(\omega, x) \\
= & {\left[\begin{array}{cccc}
0 & -P^{1}(x) & -P^{2}(x) & -P^{3}(x) \\
P^{1}(x) & 0 & M^{3}(x) & -M^{2}(x) \\
P^{2}(x) & -M^{3}(x) & 0 & M^{1}(x) \\
P^{3}(x) & M^{2}(x) & -M^{1}(x) & 0
\end{array}\right], }
\end{aligned}
$$

where $P^{1}(x), P^{2}(x), P^{3}(x)$ and $M^{1}(x), M^{2}(x), M^{3}(x)$ are the Cartesian components of the electric and magnetic polarization densities of the magneto-dielectric medium, respectively. As it is clear, the antisymmetry relations (2) give us naturally the conservation law of the total electric charges induced in the anisotropic magneto-dielectric medium. That is, we have $\partial_{\nu} J^{\nu}(x)=\partial_{\nu} \partial_{\mu} K^{\mu \nu}(x)=0$. Similarly the classical relativistic covariant Euler-Lagrange equations for the tensor fields $Y^{\alpha \beta}(\omega, x)$, modeling the magneto-dielectric medium, are

$$
\begin{aligned}
& \frac{d}{d x^{\mu}}\left(\frac{\partial £}{\partial\left(\partial_{\mu} Y_{\alpha \beta}(\omega, x)\right)}\right)-\frac{\partial £}{\partial\left(Y_{\alpha \beta}(\omega, x)\right)}=0 \\
& \quad \Rightarrow\left[-\partial_{\mu} \partial^{\mu}-\omega^{2}\right] Y^{\alpha \beta}(\omega, x)=f^{\mu \nu \alpha \beta}(\omega, x) \partial_{\mu} A_{\nu}(x) .
\end{aligned}
$$

\subsection{The relativistic covariant constitutive relation of the anisotropic magneto-dielectric medium}

The solution of the wave equation (5) consists of two parts

$$
Y^{\alpha \beta}(\omega, x)=Y_{N}^{\alpha \beta}(\omega, x)+Y_{p}^{\alpha \beta}(\omega, x) .
$$

The part $Y_{N}^{\alpha \beta}(\omega, x)$ is the solution of the homogeneous equation

$$
\left[-\partial_{\mu} \partial^{\mu}-\omega^{2}\right] Y_{N}^{\alpha \beta}(\omega, x)=0
$$

which can be written as

$$
Y_{N}^{\alpha \beta}(\omega, x)=\frac{1}{(2 \pi)^{\frac{3}{2}}} \int_{-\infty}^{+\infty} d^{3} k\left[B^{\alpha \beta}(\omega, \mathbf{k}) e^{i \mathbf{k} \cdot \mathbf{x}-i \sqrt{\omega^{2}+k^{2}} x_{0}}+\text { c.c. }\right] .
$$

After canonical quantization of the total system we replace the numerical antisymmetric constant coefficients $B^{\alpha \beta}(\omega, \mathbf{k})$ and $\left(B^{*}\right)^{\alpha \beta}(\omega, \mathbf{k})$ in (8) by suitable annihilation and creation operators, respectively. The part $Y_{p}^{\alpha \beta}(\omega, x)$ in Eq. (6) is a special solution of the wave equation (5) and is expressed as

$$
Y_{p}^{\alpha \beta}(\omega, x)=\int_{-\infty}^{+\infty} d^{4} x^{\prime} G\left(\omega, x-x^{\prime}\right) f^{\mu \nu \alpha \beta}\left(\omega, x^{\prime}\right) \partial_{\mu}^{\prime} A_{\nu}\left(x^{\prime}\right),
$$

where $G\left(\omega, x-x^{\prime}\right)$ is the retarded Green function and satisfies the equation

$$
\left[-\partial_{\mu} \partial^{\mu}-\omega^{2}\right] G\left(\omega, x-x^{\prime}\right)=\delta^{(4)}\left(x-x^{\prime}\right) .
$$




\section{Amooshahi}

Here we choose the retarded Green function for the solution of Eq. (10) in order to obtain a causal constitutive relation between the electric-magnetic polarization tensor $K^{\mu \nu}(x)$ of the magneto-dielectric medium and the strength tensor $F_{\mu \nu}(x)=$ $\partial_{\mu} A_{\nu}(x)-\partial_{\nu} A_{\mu}(x)$ of the electromagnetic field. Now substitution of the solutions (8) and (9) for the tensor fields $Y^{\alpha \beta}(\omega, x)$ in the definition of the antisymmetric electric-magnetic polarization tensor $K^{\mu \nu}(x)$ of the anisotropic magneto-dielectric medium, given by (4), gives us the relativistic covariant constitutive relation of the magneto-dielectric medium as

$$
K^{\mu \nu}(x)=K_{N}^{\mu \nu}(x)+\int_{-\infty}^{+\infty} d^{4} x^{\prime} \chi^{\mu \nu r s}\left(x, x^{\prime}\right) F_{r s}\left(x^{\prime}\right),
$$

where $F_{r s}\left(x^{\prime}\right)=\partial_{r}^{\prime} A_{s}\left(x^{\prime}\right)-\partial_{s}^{\prime} A_{r}\left(x^{\prime}\right)$ is the strength tensor of the electromagnetic field and $K_{N}^{\mu \nu}(x)$ is the noise antisymmetric electric-magnetic polarization tensor of the magneto-dielectric medium and is given by

$$
K_{N}^{\mu \nu}(x)=\frac{1}{4 \pi} \int_{0}^{\infty} d \omega f^{\mu \nu \alpha \beta}(\omega, x) Y_{N \alpha \beta}(\omega, x),
$$

where its existence is necessary for the quantization of the electromagnetic field in the presence of an absorbing magneto-dielectric medium. In constitutive relation (11) the tensor field $\chi^{\mu \nu r s}\left(x, x^{\prime}\right)$ is the susceptibility tensor of the anisotropic magneto-dielectric medium and is obtained in terms of the coupling tensor fields $f^{\mu \nu \alpha \beta}(\omega, x)$ and the retarded Green function $G\left(\omega, x-x^{\prime}\right)$ as

$$
\chi^{\mu \nu r s}\left(x, x^{\prime}\right)=\frac{1}{8 \pi} \int_{0}^{\infty} d \omega G\left(\omega, x-x^{\prime}\right) f^{\mu \nu \alpha \beta}(\omega, x) f_{\alpha \beta}^{r s}\left(\omega, x^{\prime}\right) .
$$

Using the antisymmetry relations (2) we obtain the following antisymmetry relations for the susceptibility tensor of the magneto-dielectric medium

$$
\chi^{\mu \nu r s}\left(x, x^{\prime}\right)=-\chi^{\nu \mu r s}\left(x, x^{\prime}\right), \quad \chi^{\mu \nu r s}\left(x, x^{\prime}\right)=-\chi^{\mu \nu s r}\left(x, x^{\prime}\right) .
$$

The antisymmetry relations (14) are caused the susceptibility tensor of the anisotropic magneto-dielectric medium to have 36 independent components, in Minkowski space-time. This is physically reasonable, because in the nonrelativistic electrodynamics, the electric and magnetic polarization densities of a bi-anisotropic magneto-dielectric medium, separately, are related to both the electric and magnetic fields by four susceptibility tensors of the second rank in three-dimensional usual space. These four susceptibility tensors also have totally 36 independent components in accordance to the susceptibility tensor $\chi^{\mu \nu r s}\left(x, x^{\prime}\right)$ in relativistic electrodynamics. Hence the imposition of the antisymmetry relations (2) on the coupling tensors of the electromagnetic field and the anisotropic magneto-dielectric medium completely consists with the physical and mathematical concepts of relativistic electrodynamics in the presence of anisotropic magneto-dielectric media. 


\subsection{Kramers-Kronig relations}

The memory function in any causality relation such as the constitutive relation (11) should obey the Kramers-Kronig relations that relate the real part and imaginary part of the memory function, in frequency domain, to each other. ${ }^{55}$ In this section, it is shown that, when the magneto-dielectric medium is a homogeneous one, that is when the coupling tensors $f^{\mu \nu \alpha \beta}(\omega, x)$ are independent of the space-time, the susceptibility tensor $\chi^{\mu \nu r s}$ of the medium satisfy the Kramers-Kronig relations in frequency domain. For a homogeneous anisotropic magneto-dielectric medium the susceptibility tensor of the medium defined by (13) becomes

$$
\chi^{\mu \nu r s}\left(x, x^{\prime}\right) \equiv \chi^{\mu \nu r s}\left(x-x^{\prime}\right)=\frac{1}{8 \pi} \int_{0}^{\infty} d \omega G\left(\omega, x-x^{\prime}\right) f^{\mu \nu \alpha \beta}(\omega) f_{\alpha \beta}^{r s}(\omega) .
$$

Writing the retarded Green function $G\left(\omega, x-x^{\prime}\right)$ in terms of its four-dimensional Fourier transform and using Eq. (10), one can express the susceptibility tensor of the homogeneous magneto-dielectric medium as

$$
\chi^{\mu \nu r s}\left(x-x^{\prime}\right)=\frac{1}{(8 \pi)(2 \pi)^{4}} \int_{0}^{\infty} d \omega \int_{-\infty}^{+\infty} d^{4} \kappa\left[\frac{e^{-i \kappa_{\mu}\left(x^{\mu}-x^{\prime \mu}\right)}}{\kappa_{\mu} \kappa^{\mu}-\omega^{2}} f^{\mu \nu \alpha \beta}(\omega) f^{r s}{ }_{\alpha \beta}(\omega)\right],
$$

where $\kappa=\left(\kappa_{0}, \mathbf{k}\right)$ is a point in four-dimensional reciprocal space and $x=\left(x_{0}, \mathbf{x}\right)$ is a point in Minkowski space-time. Now integrating over $\kappa_{0}$ in the complex plan $k_{0}$ on the line $k_{0}=\operatorname{Re}\left(k_{0}\right)+i 0^{+}$(in order to obtain the retarded Green function) and integrating over the angles that specify the direction of the vector $\mathbf{k}$ with respect to the vector $\mathbf{x}-\mathbf{x}^{\prime}$, we obtain the susceptibility tensor $\chi^{\mu \nu r s}\left(x-x^{\prime}\right)$ in the form

$$
\begin{aligned}
\chi^{\mu \nu r s}\left(x-x^{\prime}\right)= & -\frac{1}{16 \pi^{3}} \vartheta\left(x_{0}-x_{0}^{\prime}\right) \int_{0}^{\infty} d \alpha \sin \left[\alpha\left(x_{0}-x_{0}^{\prime}\right)\right] \int_{0}^{\alpha} d \omega \\
& \times\left[\frac{\sin \left[\sqrt{\alpha^{2}-\omega^{2}}\left|\mathbf{x}-\mathbf{x}^{\prime}\right|\right]}{\left|\mathbf{x}-\mathbf{x}^{\prime}\right|} f^{\mu \nu \alpha \beta}(\omega) f_{\alpha \beta}^{r s}(\omega)\right],
\end{aligned}
$$

where $\vartheta\left(x_{0}-x_{0}^{\prime}\right)$ is the step function. If we write the temporal Fourier transform of the susceptibility tensor of the magneto-dielectric medium as

$$
\underline{\chi}^{\mu \nu r s}\left(\omega^{\prime}, \mathbf{x}-\mathbf{x}^{\prime}\right)=\int_{-\infty}^{+\infty} d x_{0} \chi^{\mu \nu r s}\left(x_{0}, \mathbf{x}-\mathbf{x}^{\prime}\right) e^{i \omega^{\prime} x_{0}} .
$$

Then, using (17) one can obtain the real part and imaginary part of the susceptibility tensor of the magneto-dielectric medium in the real frequency domain as

$$
\begin{aligned}
\operatorname{Im}\left[\underline{\chi}^{\mu \nu r s}\left(\omega^{\prime}, \mathbf{x}-\mathbf{x}^{\prime}\right)\right]= & -\frac{1}{32 \pi^{2}} \int_{0}^{\omega^{\prime}} d \omega \\
& \times\left[\frac{\sin \left[\sqrt{\omega^{\prime 2}-\omega^{2}}\left|\mathbf{x}-\mathbf{x}^{\prime}\right|\right]}{\left|\mathbf{x}-\mathbf{x}^{\prime}\right|} f^{\mu \nu \alpha \beta}(\omega) f_{\alpha \beta}^{r s}(\omega)\right],
\end{aligned}
$$




$$
\begin{aligned}
\operatorname{Re}\left[\underline{\chi}^{\mu \nu r s}\left(\omega^{\prime}, \mathbf{x}-\mathbf{x}^{\prime}\right)\right]= & -\frac{1}{16 \pi^{3}} \int_{0}^{\infty} d \alpha \frac{\alpha}{\alpha^{2}-\omega^{\prime 2}} \int_{0}^{\alpha} d \omega \\
& \times\left[\frac{\sin \left[\sqrt{\alpha^{2}-\omega^{2}}\left|\mathbf{x}-\mathbf{x}^{\prime}\right|\right]}{\left|\mathbf{x}-\mathbf{x}^{\prime}\right|} f^{\mu \nu \alpha \beta}(\omega) f^{r s}{ }_{\alpha \beta}(\omega)\right] .
\end{aligned}
$$

An easy inspection shows that the real part and imaginary part of the susceptibility tensor of the homogeneous anisotropic magneto-dielectric medium in real frequency domain satisfy the Kramers-Kronig relations

$$
\begin{aligned}
& \operatorname{Re}\left[\underline{\chi}^{\mu \nu r s}\left(\omega^{\prime}, \mathbf{x}-\mathbf{x}^{\prime}\right)\right]=\frac{2}{\pi} P \int_{0}^{\infty} \frac{\alpha}{\alpha^{2}-\omega^{\prime 2}} \operatorname{Im}\left[\underline{\chi}^{\mu \nu r s}\left(\alpha, \mathbf{x}-\mathbf{x}^{\prime}\right)\right], \\
& \operatorname{Im}\left[\underline{\chi}^{\mu \nu r s}\left(\omega^{\prime}, \mathbf{x}-\mathbf{x}^{\prime}\right)\right]=\frac{2 \omega^{\prime}}{\pi} P \int_{0}^{\infty} d \alpha \frac{1}{\omega^{\prime 2}-\alpha^{2}} \operatorname{Re}\left[\underline{\chi}^{\mu \nu r s}\left(\alpha, \mathbf{x}-\mathbf{x}^{\prime}\right)\right],
\end{aligned}
$$

where $P$ denotes the Cauchy principal value. The Kramers-Kronig relations (21) and (22) may be found in the literatures in classical or quantum electrodynamics. ${ }^{55}$

\subsection{Canonical quantization}

In this section, a relativistic canonical quantization of both the electromagnetic field and the anisotropic polarizable and magnetizable medium is provided. Using the Lagrangian density (1) the canonical conjugate momenta of the electromagnetic field and the magneto-dielectric medium are

$$
\begin{array}{rlr}
-\Pi^{\nu}(x) & =\frac{\partial £}{\partial\left(\partial_{0} A_{\nu}(x)\right)}=-\partial_{0} A^{\nu}(x)-4 \pi K^{0 \nu}(x), & \nu=0,1,2,3, \\
Q^{\alpha \beta}(\omega, x) & =\frac{\partial £}{\partial\left(\partial_{0} Y_{\alpha \beta}(\omega, x)\right)}=\partial_{0} Y^{\alpha \beta}(\omega, x), & \alpha, \beta=0,1,2,3 .
\end{array}
$$

To quantize both the electromagnetic field and the magneto-dielectric medium we impose, in a standard way, the following equal-time commutation relations on the dynamical variables of the total system and their canonical conjugate momenta as

$$
\begin{gathered}
{\left[A^{\mu}\left(x_{0}, \mathbf{x}\right),-\Pi^{\nu}\left(x_{0}, \mathbf{x}^{\prime}\right)\right]=i \hbar g^{\mu \nu} \delta^{(3)}\left(\mathbf{x}-\mathbf{x}^{\prime}\right),} \\
{\left[Y^{\alpha \beta}\left(\omega, x_{0}, \mathbf{x}\right), Q^{\mu \nu}\left(\omega^{\prime}, x_{0}, \mathbf{x}^{\prime}\right)\right]} \\
\quad=\frac{i \hbar}{2}\left[g^{\alpha \mu} g^{\beta \nu}-g^{\alpha \nu} g^{\beta \mu}\right] \delta\left(\omega-\omega^{\prime}\right) \delta^{(3)}\left(\mathbf{x}-\mathbf{x}^{\prime}\right) .
\end{gathered}
$$

The Hamiltonian density of the total system is defined in usual way as

$$
h(x)=-\Pi^{\nu}(x) \partial_{0} A_{\nu}(x)+\int_{0}^{\infty} d \omega Q^{\alpha \beta}(\omega, x) \partial_{0} Y_{\alpha \beta}(\omega, x)-£(x) .
$$

Using the Lagrangian density (1) and the definitions of the canonical momenta of the total system given by (23) the Hamiltonian density of the total system can be 
written in terms of the dynamical fields of the total system and their canonical conjugate momenta as

$$
\begin{aligned}
h(x)= & -\frac{\left[\Pi_{\nu}(x)-4 \pi K_{0 \nu}(x)\right]\left[\Pi^{\nu}(x)-4 \pi K^{0 \nu}(x)\right]}{2} \\
& +\frac{1}{2} \partial_{l} A_{\nu}(x) \partial^{l} A^{\nu}(x)+\frac{1}{2} \int_{0}^{\infty} d \omega Q^{\alpha \beta}(\omega, x) Q_{\alpha \beta}(\omega, x) \\
& +\int_{0}^{\infty} d \omega f^{l \nu \alpha \beta}(\omega, x) \partial_{l} A_{\nu}(x) Y_{\alpha \beta}(\omega, x) \\
& -\frac{1}{2} \int_{0}^{\infty} d \omega\left[\partial_{l} Y_{\alpha \beta}(\omega, x) \partial^{l} Y^{\alpha \beta}(\omega, x)-\omega^{2} Y_{\alpha \beta}(\omega, x) Y^{\alpha \beta}(\omega, x)\right],
\end{aligned}
$$

where the summation should be done over indices $\nu, \alpha, \beta$ from 0 up to 3 and over $l$ from 1 up to 3. Applying the commutation relations (24) and using the Hamiltonian of the total system $H\left(x_{0}\right)=\int_{-\infty}^{+\infty} d^{3} x h(x)$, one can show straightforwardly, although lengthy, that the combination of the Heisenberg equations of the canonical conjugate fields $A^{\nu}(x)$ and $\Pi^{\nu}(x)$ leads to the relativistic covariant wave equation (3) for the four-vector potential in the Heisenberg picture. Also, using the commutation relations (25), the combination of the Heisenberg equations of the conjugate variables $Y^{\alpha \beta}(\omega, x)$ and $Q^{\alpha \beta}(\omega, x)$ gives us the equation of motion (5) for the tensor fields $Y^{\alpha \beta}(\omega, x)$, modeling the anisotropic magneto-dielectric medium, in Heisenberg picture. Therefore, the relativistic covariant constitutive relation (11) of the anisotropic magneto-dielectric medium is remained valid in the Heisenberg picture, where the strength tensor $F_{r s}\left(x^{\prime}\right)=\partial_{r}^{\prime} A_{s}\left(x^{\prime}\right)-\partial_{s}^{\prime} A_{r}\left(x^{\prime}\right)$ of the electromagnetic field and the noise electric-magnetic polarization tensor $K_{N}^{\mu \nu}(x)$ are Hermitian operators.

Let us introduce orthonormal four-vectors $e(\lambda, \mathbf{k}), \lambda=0,1,2,3$ for each wave vector $\mathbf{k}$ so that they obey the completeness and orthonormality relations

$$
\begin{aligned}
\sum_{\lambda=0}^{3} g_{\lambda \lambda} e^{\mu}(\lambda, \mathbf{k}) e^{\nu}(\lambda, \mathbf{k}) & =g^{\mu \nu}, \quad \mu, \nu=0,1,2,3, \\
e^{\mu}(\lambda, \mathbf{k}) e_{\mu}\left(\lambda^{\prime}, \mathbf{k}\right) & =g_{\lambda \lambda^{\prime}}, \quad \lambda, \lambda^{\prime}=0,1,2,3 .
\end{aligned}
$$

Using the four-vectors $e(\lambda, \mathbf{k}), \lambda=0,1,2,3$ we can construct the following antisymmetric tensors of the second rank for each wave vector $\mathbf{k}$ :

$$
\eta^{\alpha \beta}\left(\lambda, \lambda^{\prime}, \mathbf{k}\right)=\frac{e^{\alpha}(\lambda, \mathbf{k}) e^{\beta}\left(\lambda^{\prime}, \mathbf{k}\right)-e^{\alpha}\left(\lambda^{\prime}, \mathbf{k}\right) e^{\beta}(\lambda, \mathbf{k})}{\sqrt{2}}, \quad \lambda, \lambda^{\prime}=0,1,2,3 .
$$

Applying the completeness and orthonormality relations (28) and (29), one can show that the antisymmetric tensors introduced in the relation (30) satisfy the completeness and orthonormality relations

$$
\begin{aligned}
\sum_{\lambda, \lambda^{\prime}=0}^{3} g_{\lambda \lambda} g_{\lambda^{\prime} \lambda^{\prime}} \eta^{\alpha \beta}\left(\lambda, \lambda^{\prime}, \mathbf{k}\right) \eta^{\mu \nu}\left(\lambda, \lambda^{\prime}, \mathbf{k}\right) & =g^{\alpha \mu} g^{\beta \nu}-g^{\alpha \nu} g^{\beta \mu} \\
\eta^{\alpha \beta}\left(\lambda_{1}, \lambda_{1}^{\prime}, \mathbf{k}\right) \eta_{\alpha \beta}\left(\lambda_{2}, \lambda_{2}^{\prime}, \mathbf{k}\right) & =g_{\lambda_{1} \lambda_{2}} g_{\lambda_{1}^{\prime} \lambda_{2}^{\prime}}-g_{\lambda_{1} \lambda_{2}^{\prime}} g_{\lambda_{1}^{\prime} \lambda_{2}} .
\end{aligned}
$$




\section{Amooshahi}

Now we can expand the antisymmetric tensor fields $Y_{N}^{\alpha \beta}(\omega, x)$ introduced in (7) in terms of the antisymmetric tensors given in (30) as

$$
\begin{aligned}
Y_{N}^{\alpha \beta}(\omega, x)= & \sum_{\lambda_{1}, \lambda_{1}^{\prime}=0}^{3} \int_{-\infty}^{+\infty} d^{3} k \sqrt{\frac{\hbar}{2(2 \pi)^{3} \sqrt{k^{2}+\omega^{2}}}} \\
& \times\left[b_{\lambda_{1} \lambda_{1}^{\prime}}(\omega, \mathbf{k}) e^{i \mathbf{k} \cdot \mathbf{x}-i \sqrt{k^{2}+\omega^{2}} x_{0}}\right. \\
& \left.+b_{\lambda_{1} \lambda_{1}^{\prime}}^{\dagger}(\omega, \mathbf{k}) e^{-i \mathbf{k} \cdot \mathbf{x}+i \sqrt{k^{2}+\omega^{2}} x_{0}}\right] \eta^{\alpha \beta}\left(\lambda_{1}, \lambda_{1}^{\prime}, \mathbf{k}\right),
\end{aligned}
$$

where annihilation and creation operators $b_{\lambda_{1} \lambda_{1}^{\prime}}(\omega, \mathbf{k})$ and $b_{\lambda_{1} \lambda_{1}^{\prime}}^{\dagger}(\omega, \mathbf{k})$ satisfy the commutation relations

$$
\begin{aligned}
& {\left[b_{\lambda_{1} \lambda_{1}^{\prime}}(\omega, \mathbf{k}), b_{\lambda_{2} \lambda_{2}^{\prime}}\left(\omega^{\prime}, \mathbf{k}^{\prime}\right)\right]} \\
& \quad=\frac{1}{4}\left[g_{\lambda_{1} \lambda_{2}} g_{\lambda_{1}^{\prime} \lambda_{2}^{\prime}}-g_{\lambda_{1} \lambda_{2}^{\prime}} g_{\lambda_{1}^{\prime} \lambda_{2}}\right] \delta\left(\omega-\omega^{\prime}\right) \delta^{(3)}\left(\mathbf{k}-\mathbf{k}^{\prime}\right)
\end{aligned}
$$

compatible to the commutation relations (25). Having the expansion (33) for the tensor field $Y_{N}^{\alpha \beta}(\omega, x)$, one can write the noise antisymmetric electric-magnetic polarization tensor fields $K_{N}^{\mu \nu}(x)$ defined in (12) in terms of annihilation and creation operators $b_{\lambda_{1} \lambda_{1}^{\prime}}(\omega, \mathbf{k})$ and $b_{\lambda_{1} \lambda_{1}^{\prime}}^{\dagger}(\omega, \mathbf{k})$ of the total system (the electromagnetic field plus the anisotropic magneto-dielectric medium) as

$$
\begin{aligned}
K_{N}^{\mu \nu}(x)= & \frac{1}{4 \pi} \int_{0}^{\infty} d \omega f^{\mu \nu \alpha \beta}(\omega, x) \sum_{\lambda_{1}, \lambda_{1}^{\prime}=0}^{3} \int_{-\infty}^{+\infty} d^{3} k \sqrt{\frac{\hbar}{2(2 \pi)^{3} \sqrt{k^{2}+\omega^{2}}}} \\
& \times\left[b_{\lambda_{1} \lambda_{1}^{\prime}}(\omega, \mathbf{k}) e^{i \mathbf{k} \cdot \mathbf{x}-i \sqrt{k^{2}+\omega^{2}} x_{0}}\right. \\
& \left.+b_{\lambda_{1} \lambda_{1}^{\prime}}^{\dagger}(\omega, \mathbf{k}) e^{-i \mathbf{k} \cdot \mathbf{x}+i \sqrt{k^{2}+\omega^{2}} x_{0}}\right] \eta_{\alpha \beta}\left(\lambda_{1}, \lambda_{1}^{\prime}, \mathbf{k}\right)
\end{aligned}
$$

where will be appeared on the right-hand side of the quantum relativistic wave equation of the four-vector potential of the electromagnetic field as a source term.

\section{Anisotropic Conductor Medium}

\subsection{Relativistic classical Euler-Lagrange equations}

In this section, a relativistic canonical quantization of the electromagnetic field in the presence of an anisotropic conductor (but not polarizable and magnetizable) medium is introduced. For this purpose we model the conductor medium by a continuum set of vector fields $X^{\nu}(\omega, x)$ in Minkowski space-time labeled by a real positive parameter $\omega$. The Lagrangian density of the total system (the electromagnetic 
field plus the conductor medium) is proposed as

$$
\begin{aligned}
£(x)= & -\frac{1}{2} \partial_{\mu} A_{\nu}(x) \partial^{\mu} A^{\nu}(x)+\int_{0}^{\infty} d \omega \partial_{\mu} g^{\alpha \beta s \mu}(\omega, x) \partial_{\beta} X_{\alpha}(\omega, x) A_{s}(x) \\
& +\frac{1}{2} \int_{0}^{\infty} d \omega\left[\partial_{\mu} X_{\nu}(\omega, x) \partial^{\mu} X^{\nu}(\omega, x)-\omega^{2} X_{\nu}(\omega, x) X^{\nu}(\omega, x)\right],
\end{aligned}
$$

which is invariant under Poincaré transformations. In the Lagrangian density (36) $A^{\nu}(x)$ is the four-vector potential of the electromagnetic field and the tensor fields $g^{\alpha \beta s \mu}(\omega, x)$ are the coupling tensors that couple the electromagnetic field to the anisotropic conductor medium. We impose the antisymmetry conditions

$$
\begin{gathered}
g^{\alpha \beta \mu \nu}(\omega, x)=-g^{\alpha \beta \nu \mu}(\omega, x), \\
g^{\alpha \beta \nu \mu}(\omega, x)=-g^{\alpha \nu \beta \mu}(\omega, x), \\
g^{\nu \mu s r}(\omega, x)=-g^{\nu r s \mu}(\omega, x)
\end{gathered}
$$

on the coupling tensors $g^{\alpha \beta s \mu}(\omega, x)$. We will see that the antisymmetry relations (37) are sufficient conditions for the charge conservation principle induced in the conductor medium. Furthermore, we will see that the conductivity tensor of the anisotropic conductor medium appeared in the relativistic covariant constitutive relation of the medium is a tensor of the third rank in Minkowski space-time. The antisymmetry relation $g^{\alpha \beta \nu \mu}(\omega, x)=-g^{\alpha \nu \beta \mu}(\omega, x)$ is caused the conductivity tensor of the medium to be antisymmetric with respect to the last two indices and accordingly contains 24 independent components in Minkowski space-time. This is just the needed component numbers to relate each component of the free electric current density four-vector induced in the conductor medium to both the electric and magnetic fields. Accordingly the antisymmetry conditions (37) completely consist with the relativistic electrodynamics in the presence of an anisotropic conductor medium. The antisymmetry relation (38) is chosen in order to simplification of the calculations and beauty of the theory without disturbance of the theory in viewpoints of physical and mathematical concepts.

Using the Lagrangian density (36), one can obtain the classical relativistic covariant Euler-Lagrange equation for the four-vector potential of the electromagnetic field as

$$
\frac{d}{d x^{\mu}}\left(\frac{\partial £}{\partial\left(\partial_{\mu} A_{\nu}(x)\right)}\right)-\frac{\partial £}{\partial\left(A_{\nu}(x)\right)}=0 \Rightarrow-\partial_{\mu} \partial^{\mu} A^{\nu}(x)=\frac{4 \pi}{c} J^{\nu}(x),
$$

where

$$
J^{\nu}(x)=\frac{c}{4 \pi} \int_{0}^{\infty} d \omega \partial_{\mu} g^{\alpha \beta \nu \mu}(\omega, x) \partial_{\beta} X_{\alpha}(\omega, x)
$$

is the free electric current density four-vector induced in the anisotropic conductor medium. An easy inspection shows that the charge conservation law $\partial_{\nu} J^{\nu}(x)=0$ induced in the conductor medium is deduced using the antisymmetry relations (37). 
Also the classical relativistic covariant Euler-Lagrange equations for the vector fields $X^{\nu}(\omega, x)$, modeling the conductor medium, are obtained as

$$
\begin{aligned}
& \frac{d}{d x^{\mu}}\left(\frac{\partial £}{\partial\left(\partial_{\mu} X_{\nu}(\omega, x)\right)}\right)-\frac{\partial £}{\partial\left(X_{\nu}(\omega, x)\right)}=0 \\
& \quad \Rightarrow-\partial_{\mu} \partial^{\mu} X^{\nu}(\omega, x)-\omega^{2} X^{\nu}(\omega, x)=\partial_{r} g^{\nu \mu s r}(\omega, x) \partial_{\mu} A_{s}(x),
\end{aligned}
$$

where antisymmetry relations (38) have been used.

\subsection{The relativistic covariant constitutive relation of the anisotropic conductor medium}

In this section, the relativistic covariant constitutive relation of the anisotropic conductor medium relating the components of the free electric current density fourvector induced in the conductor medium to the strength tensor of the electromagnetic field is obtained. For this purpose, we write the solution of the wave equation (41) as two parts

$$
X^{\nu}(\omega, x)=X_{N}^{\nu}(\omega, x)+X_{p}^{\nu}(\omega, x) .
$$

The part $X_{N}^{\nu}(\omega, x)$ is the solution of the homogeneous equation

$$
\left[-\partial_{\mu} \partial^{\mu} X^{\nu}(\omega, x)-\omega^{2}\right] X_{N}^{\nu}(\omega, x)=0
$$

which can be written as

$$
X_{N}^{\nu}(\omega, x)=\frac{1}{(2 \pi)^{\frac{3}{2}}} \int_{-\infty}^{+\infty} d^{3} k\left[D^{\nu}(\omega, \mathbf{k}) e^{i \mathbf{k} \cdot \mathbf{x}-i \sqrt{\omega^{2}+k^{2}} x_{0}}+\text { c.c. }\right],
$$

where $D^{\nu}(\omega, \mathbf{k})$ and $\left(D^{*}\right)^{\nu}(\omega, \mathbf{k})$ are some numerical coefficients and will be replaced by annihilation and creation operators of the total system after canonical quantization of both the electromagnetic field and the conductor medium. The second part solution $X_{p}^{\nu}(\omega, x)$ is a special solution of the wave equation (41) which can be expressed as

$$
X_{p}^{\nu}(\omega, x)=\int_{-\infty}^{+\infty} d^{4} x^{\prime} G\left(\omega, x-x^{\prime}\right) \partial_{r}^{\prime} g^{\nu \mu s r}\left(\omega, x^{\prime}\right) \partial_{\mu}^{\prime} A_{s}\left(x^{\prime}\right),
$$

where $G\left(\omega, x-x^{\prime}\right)$ is the retarded Green function introduced by (10). Here we choose the retarded Green function in order to obtain a causal constitutive relation for the anisotropic conductor medium. Now insertion of the solution (45) and (44) into the definition of the free electric current density four-vector induced in the conductor medium given by (40) gives us the relativistic covariant constitutive relation of the anisotropic conductor medium as

$$
J^{\nu}(x)=J_{N}^{\nu}(x)+\int_{-\infty}^{+\infty} d^{4} x^{\prime} \sigma^{\nu r s}\left(x, x^{\prime}\right) F_{r s}\left(x^{\prime}\right)
$$


where $F_{r s}\left(x^{\prime}\right)=\partial_{r}^{\prime} A_{s}\left(x^{\prime}\right)-\partial_{s}^{\prime} A_{r}\left(x^{\prime}\right)$ is the strength tensor of the electromagnetic field and

$$
J_{N}^{\nu}(x)=\frac{c}{4 \pi} \int_{0}^{\infty} d \omega \partial_{\mu} g^{\alpha \beta \nu \mu}(\omega, x) \partial_{\beta} X_{N \alpha}(\omega, x)
$$

is the noise electric current density four-vector induced in the conductor medium. In the constitutive relation (46) the tensor $\sigma^{\nu r s}\left(x, x^{\prime}\right)$ is the conductivity tensor of the anisotropic conductor medium and is given by

$$
\sigma^{\nu r s}\left(x, x^{\prime}\right)=\frac{c}{8 \pi} \int_{0}^{\infty} d \omega\left[\partial_{\beta} G\left(\omega, x-x^{\prime}\right)\right] \partial_{\mu} g^{\alpha \beta \nu \mu}(\omega, x) \partial_{\delta}^{\prime} g_{\alpha}^{r s \delta}\left(\omega, x^{\prime}\right) .
$$

From the constitutive relation (46) and the definition of the conductivity tensor of the medium given by (48) it is clear that each component of the free electric current density four-vector $J^{\nu}(x)$ induced in the conductor medium is related to both the electric and magnetic fields. According to the antisymmetry relations (37) the conductivity tensor $\sigma^{\nu r s}\left(x, x^{\prime}\right)$ is antisymmetric with respect to the last two indices $r, s$. Such a tensor in four-dimensional Minkowski space-time has 24 independent components which are just the needed component numbers to relate each component of the four-vector $J^{\nu}(x)$ to both the electric and magnetic fields.

\subsection{Kramers-Kronig relations}

The conductivity tensor $\sigma^{\nu r s}\left(x, x^{\prime}\right)$ of the anisotropic conductor medium defined by (48) has been appeared in the causal relativistic covariant constitutive relation (46). Therefore, as the memory function in any causality relation should satisfy the Kramers-Kronig relations. In this section, it is shown that for a homogeneous anisotropic conductor medium, that is when the coupling tensor fields $\partial_{\mu} g^{\alpha \beta s \mu}(\omega, x) \equiv g^{\alpha \beta s}(\omega)$ is independent of the space-time, the real and imaginary parts of the conductivity tensor in real frequency domain are related to each other by the Kramers-Kronig relations and a relation other than the Kramers-Kronig relations. Using (10) and writing the retarded Green function $G\left(\omega, x-x^{\prime}\right)$ in terms of its four-dimensional Fourier transform, one can easily obtain the retarded Green function as

$$
G\left(\omega, x-x^{\prime}\right)=\frac{1}{(2 \pi)^{4}} \int_{-\infty}^{+\infty} d^{4} \kappa \frac{e^{-i \kappa_{\mu}\left(x^{\mu}-x^{\prime \mu}\right)}}{\kappa_{\mu} \kappa^{\mu}-\omega^{2}},
$$

where $x=\left(x_{0}=c t, \mathbf{x}\right)$ is a point in four-dimensional Minkowski space-time and $\kappa=\left(\kappa_{0}, \mathbf{k}\right)$ is the wave four-vector in the four-dimensional reciprocal space. Now integrating with respect to the variable $\kappa_{0}$ in the complex plan $\kappa_{0}$ on the line $\kappa_{0}=\operatorname{Re}\left(\kappa_{0}\right)+i 0^{+}$and integrating over the angles that specify the direction of the wave vector $\mathbf{k}$ with respect to $\mathbf{x}-\mathbf{x}^{\prime}$ give us the retarded Green function in a simpler form as

$$
G\left(\omega, x-x^{\prime}\right)=-\frac{\vartheta\left(x_{0}-x_{0}^{\prime}\right)}{2 \pi^{2}} \int_{0}^{\infty} k d k \frac{\sin \left[\sqrt{k^{2}+\omega^{2}}\left(x_{0}-x_{0}^{\prime}\right)\right]}{\sqrt{k^{2}+\omega^{2}}} \frac{\sin \left[k\left|\mathbf{x}-\mathbf{x}^{\prime}\right|\right]}{\left|\mathbf{x}-\mathbf{x}^{\prime}\right|},
$$




\section{Amooshahi}

where $\vartheta$ is the step function. Let us decompose the conductivity tensor of the homogeneous anisotropic conductor medium defined in (48) into two parts as

$$
\begin{aligned}
& \sigma_{0}^{\nu r s}\left(x-x^{\prime}\right)=\frac{c}{8 \pi} \int_{0}^{\infty} d \omega\left[\partial_{0} G\left(\omega, x-x^{\prime}\right)\right] g^{\alpha 0 \nu}(\omega) g_{\alpha}{ }^{r s}(\omega), \\
& \sigma_{s}^{\nu r s}\left(x-x^{\prime}\right)=\frac{c}{8 \pi} \int_{0}^{\infty} d \omega\left[\partial_{l} G\left(\omega, x-x^{\prime}\right)\right] g^{\alpha l \nu}(\omega) g_{\alpha}{ }^{r s}(\omega),
\end{aligned}
$$

where in (52) the summation should be done over $l$ from 1 up to 3 . Now substitution of the retarded Green function from (50) into (51) gives us

$$
\begin{aligned}
\sigma_{0}^{\nu r s}\left(x-x^{\prime}\right)= & -\frac{c \vartheta\left(x_{0}-x_{0}^{\prime}\right)}{16 \pi^{3}} \int_{0}^{\infty} d \alpha \alpha \cos \left[\alpha\left(x_{0}-x_{0}^{\prime}\right)\right] \int_{0}^{\alpha} d \omega \\
& \times\left[\frac{\sin \left[\sqrt{\alpha^{2}-\omega^{2}}\left|\mathbf{x}-\mathbf{x}^{\prime}\right|\right]}{\left|\mathbf{x}-\mathbf{x}^{\prime}\right|} g^{\alpha 0 \nu}(\omega) g_{\alpha}{ }^{r s}(\omega)\right] .
\end{aligned}
$$

Also by substitution of the retarded Green function from (50) into (52) we deduce

$$
\begin{aligned}
\sigma_{s}^{\nu r s}\left(x-x^{\prime}\right)= & -\frac{c \vartheta\left(x_{0}-x_{0}^{\prime}\right)}{16 \pi^{3}} \int_{0}^{\infty} d \alpha \sin \left[\alpha\left(x_{0}-x_{0}^{\prime}\right)\right] \int_{0}^{\alpha} d \omega \\
& \times\left[\partial_{l}\left(\frac{\sin \left[\sqrt{\alpha^{2}-\omega^{2}}\left|\mathbf{x}-\mathbf{x}^{\prime}\right|\right]}{\left|\mathbf{x}-\mathbf{x}^{\prime}\right|}\right) g^{\alpha l \nu}(\omega) g_{\alpha}{ }^{r s}(\omega)\right] .
\end{aligned}
$$

Using the expressions (53) and (54) it can be easily shown that the real and imaginary parts of $\sigma_{0}^{\nu r s}\left(x-x^{\prime}\right)$ and $\sigma_{s}^{\nu r s}\left(x-x^{\prime}\right)$ in real frequency domain are

$$
\begin{aligned}
& \operatorname{Re}\left[\underline{\sigma_{0}^{\nu r s}}\left(\omega^{\prime}, \mathbf{x}-\mathbf{x}^{\prime}\right)\right] \\
& =-\frac{c \omega^{\prime}}{32 \pi^{2}} \int_{0}^{\omega^{\prime}} d \omega\left[\frac{\sin \left[\sqrt{\omega^{\prime 2}-\omega^{2}}\left|\mathbf{x}-\mathbf{x}^{\prime}\right|\right]}{\left|\mathbf{x}-\mathbf{x}^{\prime}\right|} g^{\alpha 0 \nu}(\omega) g_{\alpha}{ }^{r s}(\omega)\right] \\
& \operatorname{Im}\left[\underline{\sigma_{0}^{\nu r s}}\left(\omega^{\prime}, \mathbf{x}-\mathbf{x}^{\prime}\right)\right] \\
& =\frac{c \omega^{\prime}}{16 \pi^{3}} \int_{0}^{\infty} d \alpha \int_{0}^{\alpha} d \omega \frac{\alpha}{\alpha^{2}-\omega^{\prime 2}} \frac{\sin \left[\sqrt{\alpha^{2}-\omega^{2}}\left|\mathbf{x}-\mathbf{x}^{\prime}\right|\right]}{\left|\mathbf{x}-\mathbf{x}^{\prime}\right|} g^{\alpha 0 \nu}(\omega) g_{\alpha}{ }^{r s}(\omega), \\
& \operatorname{Re}\left[{\underline{\sigma_{s}}}^{\nu r s}\left(\omega^{\prime}, \mathbf{x}-\mathbf{x}^{\prime}\right)\right] \\
& =-\frac{c}{16 \pi^{3}} \int_{0}^{\infty} d \alpha \int_{0}^{\alpha} d \omega \frac{\alpha}{\alpha^{2}-\omega^{\prime 2}}\left[\partial_{l}\left(\frac{\sin \left[\sqrt{\alpha^{2}-\omega^{2}}\left|\mathbf{x}-\mathbf{x}^{\prime}\right|\right]}{\left|\mathbf{x}-\mathbf{x}^{\prime}\right|}\right) g^{\alpha l \nu}(\omega) g_{\alpha}{ }^{r s}(\omega)\right] \\
& \operatorname{Im}\left[{\underline{\sigma_{s}}}^{\nu r s}\left(\omega^{\prime}, \mathbf{x}-\mathbf{x}^{\prime}\right)\right] \\
& =-\frac{c}{32 \pi^{2}} \int_{0}^{\omega^{\prime}} d \omega\left[\partial_{l}\left(\frac{\sin \left[\sqrt{\omega^{\prime 2}-\omega^{2}}\left|\mathbf{x}-\mathbf{x}^{\prime}\right|\right]}{\left|\mathbf{x}-\mathbf{x}^{\prime}\right|}\right) g^{\alpha l \nu}(\omega) g_{\alpha}{ }^{r s}(\omega)\right]
\end{aligned}
$$


where ${\underline{\sigma_{0}}}^{\nu r s}\left(\omega^{\prime}, \mathbf{x}-\mathbf{x}^{\prime}\right)$ and ${\underline{\sigma_{s}}}^{\nu r s}\left(\omega^{\prime}, \mathbf{x}-\mathbf{x}^{\prime}\right)$ are the temporal Fourier transforms of $\left.\sigma_{0}^{\nu r s} \overline{(x}-x^{\prime}\right)$ and $\sigma_{s}^{\nu r s}\left(x-\overline{x^{\prime}}\right)$, respectively and are defined by

$$
\begin{aligned}
& {\underline{\sigma_{0}}}^{\nu r s}\left(\omega^{\prime}, \mathbf{x}-\mathbf{x}^{\prime}\right)=\int_{-\infty}^{+\infty} d x_{0} \sigma_{0}^{\nu r s}\left(x_{0}, \mathbf{x}-\mathbf{x}^{\prime}\right) e^{i \omega^{\prime} x_{0}} \\
& {\underline{\sigma_{s}}}^{\nu r s}\left(\omega^{\prime}, \mathbf{x}-\mathbf{x}^{\prime}\right)=\int_{-\infty}^{+\infty} d x_{0} \sigma_{s}^{\nu r s}\left(x_{0}, \mathbf{x}-\mathbf{x}^{\prime}\right) e^{i \omega^{\prime} x_{0}} .
\end{aligned}
$$

An easy inspection shows that the real and imaginary parts of the $\underline{\sigma}_{s}{ }^{\nu r s}\left(\omega^{\prime}, \mathbf{x}-\mathbf{x}^{\prime}\right)$ given by (57) and (58) satisfy the Kramers-Kronig relations

$$
\begin{aligned}
& \operatorname{Re}\left[{\underline{\sigma_{s}}}^{\nu r s}\left(\omega^{\prime}, \mathbf{x}-\mathbf{x}^{\prime}\right)\right]=\frac{2}{\pi} P \int_{0}^{\infty} d \alpha \frac{\alpha}{\alpha^{2}-\omega^{\prime 2}} \operatorname{Im}\left[{\underline{\sigma_{s}}}^{\nu r s}\left(\alpha, \mathbf{x}-\mathbf{x}^{\prime}\right)\right] \\
& \operatorname{Im}\left[{\underline{\sigma_{s}}}^{\nu r s}\left(\omega^{\prime}, \mathbf{x}-\mathbf{x}^{\prime}\right)\right]=-\frac{2 \omega^{\prime}}{\pi} P \int_{0}^{\infty} d \alpha \frac{1}{\alpha^{2}-\omega^{\prime 2}} \operatorname{Re}\left[{\underline{\sigma_{s}}}^{\nu r s}\left(\alpha, \mathbf{x}-\mathbf{x}^{\prime}\right)\right]
\end{aligned}
$$

in real frequency domain which are the same as the Kramers-Kronig relations for the susceptibility tensor of an anisotropic magneto-dielectric medium given by (21) and (22). It can be easily shown that the real and imaginary parts of ${\underline{\sigma_{0}}}^{\nu r s}\left(\omega^{\prime}, \mathbf{x}-\mathbf{x}^{\prime}\right)$ are related to each other by the relations

$$
\begin{aligned}
& \operatorname{Re}\left[{\underline{\sigma_{0}}}^{\nu r s}\left(\omega^{\prime}, \mathbf{x}-\mathbf{x}^{\prime}\right)\right]=\frac{2 \omega^{\prime}}{\pi} P \int_{0}^{\infty} d \alpha \frac{1}{\alpha^{2}-\omega^{\prime 2}} \operatorname{Im}\left[{\underline{\sigma_{0}}}^{\nu r s}\left(\alpha, \mathbf{x}-\mathbf{x}^{\prime}\right)\right] \\
& \operatorname{Im}\left[{\underline{\sigma_{0}}}^{\nu r s}\left(\omega^{\prime}, \mathbf{x}-\mathbf{x}^{\prime}\right)\right]=-\frac{2 \omega^{\prime}}{\pi} P \int_{0}^{\infty} d \alpha \frac{1}{\alpha^{2}-\omega^{\prime 2}} \operatorname{Re}\left[{\underline{\sigma_{0}}}^{\nu r s}\left(\alpha, \mathbf{x}-\mathbf{x}^{\prime}\right)\right]
\end{aligned}
$$

in real frequency domain. The relations $(62)$ for ${\underline{\sigma_{0}}}^{\nu r s}\left(\omega^{\prime}, \mathbf{x}-\mathbf{x}^{\prime}\right)$ are different from the Kramers-Kronig relations (61), (21) and (22) which are found in the litera-

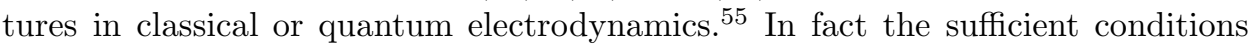
to establish the Kramers-Kronig relations (61) in real frequency domain for the memory function of a causal relation are:

(1) The temporal Fourier transform of the memory function should be an analytical complex function on the semi-complex plan $\operatorname{Im}\left(\omega^{\prime}\right) \geq 0$.

(2) The temporal Fourier transform of the memory function should be fall to zero as or faster than $\frac{1}{\omega^{\prime}}$ on the semi-complex plan $\operatorname{Im}\left(\omega^{\prime}\right) \geq 0$ when $\left|\omega^{\prime}\right|$ tends to infinity.

From the relation (55) the asymptotic form of the $\operatorname{Re}\left[\underline{\sigma_{0}^{\nu r s}}\left(\omega^{\prime}, \mathbf{x}-\mathbf{x}^{\prime}\right)\right]$ for very large real frequencies is

$$
\operatorname{Re}\left[\underline{\sigma_{0}^{\nu r s}}\left(\omega^{\prime}, \mathbf{x}-\mathbf{x}^{\prime}\right)\right] \simeq-\frac{c \omega^{\prime}}{32 \pi^{2}} \frac{\sin \left[\omega^{\prime}\left|\mathbf{x}-\mathbf{x}^{\prime}\right|\right]}{\left|\mathbf{x}-\mathbf{x}^{\prime}\right|} \int_{0}^{\infty} d \omega\left[g^{\alpha 0 \nu}(\omega) g_{\alpha}{ }^{r s}(\omega)\right],
$$

which does not fall to zero provided that the integral

$$
\int_{0}^{\infty} d \omega g^{\alpha 0 \nu}(\omega) g_{\alpha}{ }^{r s}(\omega)
$$




\section{Amooshahi}

to be have a nonzero value. Therefore, $\sigma_{0}^{\nu r s}\left(\omega^{\prime}, \mathbf{x}-\mathbf{x}^{\prime}\right)$ is not an analytical complex function on the real axis of the complex plane $\omega^{\prime}$. Accordingly $\sigma_{0}^{\nu r s}\left(\omega^{\prime}, \mathbf{x}-\mathbf{x}^{\prime}\right)$ as a complex function of the complex frequency $\omega^{\prime}$ does not have the sufficient conditions (1) and (2) mentioned above to satisfy the Kramers-Kronig relations.

\subsection{Canonical quantization}

We are now ready to provide a canonical relativistic quantization for both the electromagnetic fields and the anisotropic conductor medium using the Lagrangian density (36). The canonical conjugate momenta of the dynamical fields $A^{\nu}(x)$ and $X^{\nu}(\omega, x)$ in an standard way are defined by

$$
\begin{aligned}
-\Pi^{\nu}(x) & =\frac{\partial £}{\partial\left(\partial_{0} A_{\nu}(x)\right)}=-\partial^{0} A^{\nu}(x), \\
D^{\nu}(\omega, x) & =\frac{\partial £}{\partial\left(\partial_{0} X_{\nu}(\omega, x)\right)}=\partial^{0} X^{\nu}(\omega, x)+\partial_{\mu} g^{\nu 0 s \mu}(\omega, x) A_{s}(x) .
\end{aligned}
$$

Canonical quantization of both the electromagnetic field and the anisotropic conductor medium can be achieved by imposing the standard equal-time commutation relations on the conjugate variable pairs $A^{\mu}(x),-\Pi^{\nu}(x)$ and $X^{\mu}(\omega, x), D^{\nu}(\omega, x)$ as

$$
\begin{aligned}
{\left[A^{\mu}\left(x_{0}, \mathbf{x}\right),-\Pi^{\nu}\left(x_{0}, \mathbf{x}^{\prime}\right)\right] } & =i \hbar g^{\mu \nu} \delta^{(3)}\left(\mathbf{x}-\mathbf{x}^{\prime}\right), \\
{\left[X^{\mu}\left(\omega, x_{0}, \mathbf{x}\right), D^{\nu}\left(\omega^{\prime}, x_{0}, \mathbf{x}^{\prime}\right)\right] } & =i \hbar g^{\mu \nu} \delta^{(3)}\left(\mathbf{x}-\mathbf{x}^{\prime}\right) \delta\left(\omega-\omega^{\prime}\right) .
\end{aligned}
$$

The Hamiltonian density of the total system (the electromagnetic field plus the anisotropic conductor medium) is expressed as

$$
h(x)=-\Pi^{\nu}(x) \partial_{0} A_{\nu}(x)+\int_{0}^{\infty} d \omega D^{\nu}(\omega, x) \partial_{0} X_{\nu}(\omega, x)-£(x),
$$

where after eliminating the derivatives $\partial_{0} A_{\nu}(x)$ and $\partial_{0} X_{\nu}(\omega, x)$, using the definitions (65) and (66), the Hamiltonian density $h(x)$ can be written in terms of the conjugate variable pairs $A^{\mu}(x),-\Pi^{\nu}(x)$ and $X^{\mu}(\omega, x), D^{\nu}(\omega, x)$ as

$$
\begin{aligned}
h(x)= & -\frac{1}{2} \Pi_{\nu}(x) \Pi^{\nu}(x)+\frac{1}{2} \partial_{l} A_{\nu}(x) \partial^{l} A^{\nu}(x) \\
& +\frac{1}{2} \int_{0}^{\infty} d \omega\left[\left(D^{\alpha}(\omega, x)-\partial_{\mu} g^{\alpha 0 s \mu}(\omega, x) A_{s}(x)\right)\right. \\
& \left.\times\left(D_{\alpha}(\omega, x)-\partial^{\mu} g_{\alpha 0 r \mu}(\omega, x) A^{r}(x)\right)\right] \\
& -\int_{0}^{\infty} d \omega \partial_{\mu} g^{\alpha l s \mu}(\omega, x) \partial_{l} X_{\alpha}(\omega, x) A_{s}(x) \\
& -\frac{1}{2} \int_{0}^{\infty} d \omega\left[\partial_{l} X_{\nu}(\omega, x) \partial^{l} X^{\nu}(\omega, x)-\omega^{2} X_{\nu}(\omega, x) X^{\nu}(\omega, x)\right],
\end{aligned}
$$


where the summation should be done over the indices $\nu, \alpha, \mu, s, r$ from 0 up to 3 and the over $l$ from 1 up to 3 . Using the Hamiltonian $H\left(x_{0}\right)=\int d^{3} x h(x)$ of the total system (the electromagnetic field plus the conductor medium) and applying the commutation relation (67), a straightforward but relatively lengthy calculation shows that the combination of the equations of motion of the conjugate variable pairs $-\Pi^{\nu}(x)$ and $A^{\nu}(x)$ in the Heisenberg picture leads to the relativistic covariant wave equation (39) for the potential four-vector of the electromagnetic field, where the electric current density four-vector is defined by (40). Also, straightforwardly, it can be shown that the combination of the Heisenberg equations of the conjugate variable pairs $D^{\nu}(\omega, x)$ and $X^{\nu}(\omega, x)$ gives us the relativistic covariant equation of motion (41) for the vector fields $X^{\nu}(\omega, x)$ modeling the conductor medium. Therefore, the relativistic covariant constitutive relation of the anisotropic conductor medium given by (46) is remained valid in the quantum electrodynamics, where now the free electric current density four-vector $J^{\nu}(x)$ induced in the conductor medium and the strength tensor of the electromagnetic field $F_{r s}\left(x^{\prime}\right)=\partial_{r}^{\prime} A_{s}\left(x^{\prime}\right)-\partial_{s}^{\prime} A_{r}\left(x^{\prime}\right)$ are Hermitian operators.

One can expand the first part of the solution (42) of the wave equation (41) in terms of the plane waves as

$$
\begin{aligned}
X_{N}^{\nu}(\omega, x)= & \sum_{\lambda=0}^{3} \int_{-\infty}^{+\infty} d^{3} k \sqrt{\frac{\hbar}{2(2 \pi)^{3} \sqrt{k^{2}+\omega^{2}}}} \\
& \times\left[a_{\lambda}(\omega, \mathbf{k}) e^{i \mathbf{k} \cdot \mathbf{x}-i \sqrt{k^{2}+\omega^{2}} x_{0}}+a_{\lambda}^{\dagger}(\omega, \mathbf{k}) e^{-i \mathbf{k} \cdot \mathbf{x}+i \sqrt{k^{2}+\omega^{2}} x_{0}}\right] e^{\nu}(\lambda, \mathbf{k}),
\end{aligned}
$$

where $e^{\nu}(\lambda, \mathbf{k}), \lambda=0,1,2,3$ for each wave vector $\mathbf{k}$ are the four-vectors that satisfy the completeness and orthonormality relations (28) and (29) in Minkowski spacetime. In (71) the annihilation and creation operators $a_{\lambda}(\omega, \mathbf{k}), a_{\lambda}^{\dagger}(\omega, \mathbf{k})$ satisfy the commutation relations

$$
\left[a_{\lambda}(\omega, \mathbf{k}), a_{\lambda^{\prime}}^{\dagger}\left(\omega^{\prime}, \mathbf{k}^{\prime}\right)\right]=g_{\lambda \lambda^{\prime}} \delta^{(3)}\left(\mathbf{k}-\mathbf{k}^{\prime}\right) \delta\left(\omega-\omega^{\prime}\right)
$$

in compatible to the commutation relations (68). Hence the noise electric current density four-vector $J_{N}^{\nu}(x)$ in the constitutive relation (46) of the conductor medium given by (47) can be expressed in terms of the annihilation and creation operators of the total system (the electromagnetic field plus the anisotropic conductor medium) $a_{\lambda}(\omega, \mathbf{k}), a_{\lambda}^{\dagger}(\omega, \mathbf{k})$ as

$$
\begin{aligned}
J_{N}^{\nu}(x)= & \frac{c}{4 \pi} \int_{0}^{\infty} d \omega \partial_{\mu} g^{\alpha \beta \nu \mu}(\omega, x) \partial_{\beta} X_{N \alpha}(\omega, x) \\
= & \frac{c}{4 \pi} \int_{0}^{\infty} d \omega \partial_{\mu} g^{\alpha \beta \nu \mu}(\omega, x) \sum_{\lambda=0}^{3} \int_{-\infty}^{+\infty} d^{4} \kappa \sqrt{\frac{\hbar}{2(2 \pi)^{3} \sqrt{k^{2}+\omega^{2}}}} \\
& \times \delta\left(\kappa^{0}-\sqrt{k^{2}+\omega^{2}}\right)\left[\left(-i \kappa_{\beta}\right) a_{\lambda}(\omega, \mathbf{k}) e^{-i \kappa_{\beta} x^{\beta}}+\text { h.c. }\right] e_{\alpha}(\lambda, \mathbf{k}),
\end{aligned}
$$


where $k=|\mathbf{k}|, x=\left(x_{0}=c t, \mathbf{x}\right)$ is a point in four-dimensional Minkowski spacetime and $\kappa=\left(\kappa_{0}, \mathbf{k}\right)$ is a point in the four-dimensional reciprocal space. In the next section we will see that the noise electric current density four-vector given by (73) will be appeared on the right-hand side of the quantum relativistic wave equation of the four-vector potential of the electromagnetic field as a source term.

\section{Anisotropic Conductor Polarizable and Magnetizable Medium}

\subsection{Relativistic classical Euler-Lagrange equation}

In order to quantize electromagnetic field in the presence of an anisotropic conductor polarizable and magnetizable medium in a canonical relativistic language, following the two previous sections, we model the medium by a continuum set of antisymmetric tensor fields $Y^{\alpha \beta}(\omega, x)$ and a continuum set of vector fields $X^{\nu}(\omega, x)$ in Minkowski space-time each is labeled by a positive real parameter $\omega$. The continuum set of the antisymmetric tensor fields $Y^{\alpha \beta}(\omega, x)$ describes the polarization and the magnetization properties of the medium and the continuum set of the vector fields $X^{\nu}(\omega, x)$ describes the conductivity property of the medium. The reason of the choice of a continuum set of the antisymmetric tensor fields $Y^{\alpha \beta}(\omega, x)$ to describe the polarization and the magnetization properties of the medium has been explained previously in Sec. 2. The Lagrangian density of the total system (the electromagnetic field plus the anisotropic conductor polarizable and magnetizable medium) is proposed as

$$
\begin{aligned}
£(x)= & -\frac{1}{2} \partial_{\mu} A_{\nu}(x) \partial^{\mu} A^{\nu}(x)-\int_{0}^{\infty} d \omega f^{\mu \nu \alpha \beta}(\omega, x) \partial_{\mu} A_{\nu}(x) Y_{\alpha \beta}(\omega, x) \\
& +\int_{0}^{\infty} d \omega \partial_{\mu} g^{\alpha \beta s \mu}(\omega, x) \partial_{\beta} X_{\alpha}(\omega, x) A_{s}(x) \\
& +\frac{1}{2} \int_{0}^{\infty} d \omega\left[\partial_{\mu} Y_{\alpha \beta}(\omega, x) \partial^{\mu} Y^{\alpha \beta}(\omega, x)-\omega^{2} Y_{\alpha \beta}(\omega, x) Y^{\alpha \beta}(\omega, x)\right] \\
& +\frac{1}{2} \int_{0}^{\infty} d \omega\left[\partial_{\mu} X_{\nu}(\omega, x) \partial^{\mu} X^{\nu}(\omega, x)-\omega^{2} X_{\nu}(\omega, x) X^{\nu}(\omega, x)\right] .
\end{aligned}
$$

The tensors fields $f^{\mu \nu \alpha \beta}(\omega, x)$ and $g^{\alpha \beta s \mu}(\omega, x)$ are the coupling tensors of the electromagnetic field and the anisotropic conductor magneto-dielectric medium and obey the antisymmetry relations (2), (37) and (38). The reason of imposing these antisymmetry relations on the coupling tensor fields $f^{\mu \nu \alpha \beta}(\omega, x)$ and $g^{\alpha \beta s \mu}(\omega, x)$ was mentioned in detail in Secs. 2 and $\underline{3}$. The classical relativistic covariant EulerLagrange equation of the four-vector potential of the electromagnetic field can be easily written as

$$
\begin{aligned}
& \frac{d}{d x^{\mu}}\left(\frac{\partial £}{\partial\left(\partial_{\mu} A_{\nu}(x)\right)}\right)-\frac{\partial £}{\partial\left(A_{\nu}(x)\right)}=0 \\
& \quad \Rightarrow-\partial_{\mu} \partial^{\mu} A^{\nu}(x)=4 \pi \partial_{\mu} K^{\mu \nu}(x)+\frac{4 \pi}{c} J^{\nu}(x),
\end{aligned}
$$


where $K^{\mu \nu}(x)$ and $J^{\nu}(x)$ are the antisymmetric electric-magnetic polarization tensor field of the medium and the free electric current density four-vector induced in the medium, respectively and are defined by (4) and (40). The antisymmetry conditions (2) and (37) on the coupling tensors of the electromagnetic field and the anisotropic conductor magneto-dielectric medium give us the conservation law of the total charges induced in the medium.

The classical relativistic covariant Euler-Lagrange equations of the antisymmetric tensor fields $Y^{\alpha \beta}(\omega, x)$ and the vector fields $X^{\nu}(\omega, x)$, modeling the anisotropic conductor magneto-dielectric medium, are

$$
\begin{aligned}
& \frac{d}{d x^{\mu}}\left(\frac{\partial £}{\partial\left(\partial_{\mu} Y_{\alpha \beta}(\omega, x)\right)}\right)-\frac{\partial £}{\partial\left(Y_{\alpha \beta}(\omega, x)\right)}=0 \\
& \quad \Rightarrow\left[-\partial_{\mu} \partial^{\mu}-\omega^{2}\right] Y^{\alpha \beta}(\omega, x)=f^{\mu \nu \alpha \beta}(\omega, x) \partial_{\mu} A_{\nu}(x), \\
& \frac{d}{d x^{\mu}}\left(\frac{\partial £}{\partial\left(\partial_{\mu} X_{\nu}(\omega, x)\right)}\right)-\frac{\partial £}{\partial\left(X_{\nu}(\omega, x)\right)}=0 \\
& \quad \Rightarrow-\partial_{\mu} \partial^{\mu} X^{\nu}(\omega, x)-\omega^{2} X^{\nu}(\omega, x)=\partial_{r} g^{\nu \mu s r}(\omega, x) \partial_{\mu} A_{s}(x) .
\end{aligned}
$$

\subsection{The relativistic covariant constitutive relations of the anisotropic conductor magneto-dielectric medium}

The solutions of the wave equations (76) and (77) can be written as two parts

$$
\begin{gathered}
Y^{\alpha \beta}(\omega, x)=Y_{N}^{\alpha \beta}(\omega, x)+Y_{p}^{\alpha \beta}(\omega, x), \\
X^{\nu}(\omega, x)=X_{N}^{\nu}(\omega, x)+X_{p}^{\nu}(\omega, x)
\end{gathered}
$$

as the previous two sections. The first parts $Y_{N}^{\alpha \beta}(\omega, x)$ and $X_{N}^{\nu}(\omega, x)$ are the solution of the homogeneous equations

$$
\begin{aligned}
{\left[-\partial_{\mu} \partial^{\mu}-\omega^{2}\right] Y_{N}^{\alpha \beta}(\omega, x) } & =0, \\
{\left[-\partial_{\mu} \partial^{\mu} X^{\nu}(\omega, x)-\omega^{2}\right] X_{N}^{\nu}(\omega, x) } & =0
\end{aligned}
$$

and are given by (8) and (44). The second parts of the solutions $Y_{p}^{\alpha \beta}(\omega, x)$ and $X_{p}^{\nu}(\omega, x)$ in $(78)$ and $(79)$ are the special solutions of the relativistic wave equations (76) and (77), respectively and are expressed by (9) and (45). Substitutions of the solutions (78) in (4) and (79) in (40) and using the expressions (9) and (45) lead to the relativistic covariant constitutive relations of the anisotropic conductor magneto-dielectric medium relating the antisymmetric electric-magnetic polarization tensor of the medium and the free electric current density four-vector, induced in the medium, to the strength tensor of the electromagnetic field as

$$
\begin{gathered}
K^{\mu \nu}(x)=K_{N}^{\mu \nu}(x)+\int_{-\infty}^{+\infty} d^{4} x^{\prime} \chi^{\mu \nu r s}\left(x, x^{\prime}\right) F_{r s}\left(x^{\prime}\right), \\
J^{\nu}(x)=J_{N}^{\nu}(x)+\int_{-\infty}^{+\infty} d^{4} x^{\prime} \sigma^{\nu r s}\left(x, x^{\prime}\right) F_{r s}\left(x^{\prime}\right),
\end{gathered}
$$




\section{Amooshahi}

where $K_{N}^{\mu \nu}(x)$ and $J_{N}^{\nu}(x)$ are the noise electric-magnetic polarization tensor and the noise free electric current density four-vector, respectively and given by (12) and (47). In the constitutive relations (82) and (83) the tensors $\chi^{\mu \nu r s}\left(x, x^{\prime}\right)$ and $\sigma^{\nu r s}\left(x, x^{\prime}\right)$ are the susceptibility and the conductivity tensors of the anisotropic conductor magneto-dielectric medium, respectively and are expressed as

$$
\begin{aligned}
\chi^{\mu \nu r s}\left(x, x^{\prime}\right) & =\frac{1}{8 \pi} \int_{0}^{\infty} d \omega G\left(\omega, x-x^{\prime}\right) f^{\mu \nu \alpha \beta}(\omega, x) f_{\alpha \beta}^{r s}\left(\omega, x^{\prime}\right), \\
\sigma^{\nu r s}\left(x, x^{\prime}\right) & =\frac{c}{8 \pi} \int_{0}^{\infty} d \omega\left[\partial_{\beta} G\left(\omega, x-x^{\prime}\right)\right] \partial_{\mu} g^{\alpha \beta \nu \mu}(\omega, x) \partial_{\delta}^{\prime} g_{\alpha}{ }^{r s \delta}\left(\omega, x^{\prime}\right),
\end{aligned}
$$

where are coincided (13) and (48) and satisfy the antisymmetry relations

$$
\begin{aligned}
\chi^{\mu \nu r s}\left(x, x^{\prime}\right) & =-\chi^{\nu \mu r s}\left(x, x^{\prime}\right), \\
\chi^{\mu \nu r s}\left(x, x^{\prime}\right) & =-\chi^{\mu \nu s r}\left(x, x^{\prime}\right), \\
\sigma^{\nu r s}\left(x, x^{\prime}\right) & =-\sigma^{\nu s r}\left(x, x^{\prime}\right) .
\end{aligned}
$$

As it was shown in the previous two sections the susceptibility tensor $\chi^{\mu \nu r s}\left(x, x^{\prime}\right)$ and the conductivity tensor $\sigma^{\nu r s}\left(x, x^{\prime}\right)$ obey the Kramers-Kronig relations (21), (22), (61) and the relation (62) in real frequency domain for the homogeneous anisotropic bulk medium.

\subsection{Canonical quantization}

Having the Lagrangian density (74) one can provide a relativistic canonical quantization of both the electromagnetic field and the anisotropic conductor polarizable and magnetizable medium. For this purpose the canonical conjugate momenta of the total system are defined in the usual way as

$$
\begin{aligned}
-\Pi^{\nu}(x) & =\frac{\partial £}{\partial\left(\partial_{0} A_{\nu}(x)\right)}=-\partial^{0} A^{\nu}(x)-K^{0 \nu}(x) \\
D^{\nu}(\omega, x) & =\frac{\partial £}{\partial\left(\partial_{0}\left(X_{\nu}(x)\right)\right)}=\partial^{0} X^{\nu}(\omega, x)+\partial_{\mu} g^{\nu 0 s \mu}(\omega, x) A_{s}(x), \\
Q^{\alpha \beta}(\omega, x) & =\frac{\partial £}{\partial\left(\partial_{0} Y_{\alpha \beta}(\omega, x)\right)}=\partial^{0} Y^{\alpha \beta}(\omega, x) .
\end{aligned}
$$

To quantize the total system, we impose the equal-time commutation relations

$$
\begin{gathered}
{\left[A^{\mu}\left(x_{0}, \mathbf{x}\right),-\Pi^{\nu}\left(x_{0}, \mathbf{x}^{\prime}\right)\right]=i \hbar g^{\mu \nu} \delta^{(3)}\left(\mathbf{x}-\mathbf{x}^{\prime}\right),} \\
{\left[Y^{\alpha \beta}\left(\omega, x_{0}, \mathbf{x}\right), Q^{\mu \nu}\left(\omega^{\prime}, x_{0}, \mathbf{x}^{\prime}\right)\right]} \\
=\frac{i \hbar}{2}\left[g^{\alpha \mu} g^{\beta \nu}-g^{\alpha \nu} g^{\beta \mu}\right] \delta\left(\omega-\omega^{\prime}\right) \delta^{(3)}\left(\mathbf{x}-\mathbf{x}^{\prime}\right), \\
{\left[X^{\mu}\left(\omega, x_{0}, \mathbf{x}\right), D^{\nu}\left(\omega^{\prime}, x_{0}, \mathbf{x}^{\prime}\right)\right]=i \hbar g^{\mu \nu} \delta^{(3)}\left(\mathbf{x}-\mathbf{x}^{\prime}\right) \delta\left(\omega-\omega^{\prime}\right)}
\end{gathered}
$$


on the dynamical fields of the system and their canonical conjugate momenta in a standard way. The Hamiltonian density of the total system (the electromagnetic field plus the anisotropic conductor magneto-dielectric medium) can be written as

$$
\begin{aligned}
h(x)= & -\Pi^{\nu}(x) \partial_{0} A_{\nu}(x)+\int_{0}^{\infty} d \omega Q^{\alpha \beta}(\omega, x) \partial_{0} Y_{\alpha \beta}(\omega, x) \\
& +\int_{0}^{\infty} d \omega D^{\nu}(\omega, x) \partial_{0} X_{\nu}(\omega, x)-£(x) .
\end{aligned}
$$

After eliminating the derivatives $\partial_{0} A_{\nu}(x), \partial_{0} Y_{\alpha \beta}(\omega, x), \partial_{0} X_{\nu}(\omega, x)$, using definitions (87), the Hamiltonian density of the total system can be expressed in terms of the dynamical fields of the system and their canonical conjugate momenta as

$$
\begin{aligned}
h(x)= & -\frac{\left[\Pi_{\nu}(x)-4 \pi K_{0 \nu}(x)\right]\left[\Pi^{\nu}(x)-4 \pi K^{0 \nu}(x)\right]}{2} \\
& +\frac{1}{2} \partial_{l} A_{\nu}(x) \partial^{l} A^{\nu}(x)+\frac{1}{2} \int_{0}^{\infty} d \omega Q^{\alpha \beta}(\omega, x) Q_{\alpha \beta}(\omega, x) \\
& +\int_{0}^{\infty} d \omega f^{l \nu \alpha \beta}(\omega, x) \partial_{l} A_{\nu}(x) Y_{\alpha \beta}(\omega, x) \\
& -\frac{1}{2} \int_{0}^{\infty} d \omega\left[\partial_{l} Y_{\alpha \beta}(\omega, x) \partial^{l} Y^{\alpha \beta}(\omega, x)-\omega^{2} Y_{\alpha \beta}(\omega, x) Y^{\alpha \beta}(\omega, x)\right] \\
& +\frac{1}{2} \int_{0}^{\infty} d \omega\left[\left(D^{\alpha}(\omega, x)-\partial_{\mu} g^{\alpha 0 s \mu}(\omega, x) A_{s}(x)\right)\right. \\
& \left.\times\left(D_{\alpha}(\omega, x)-\partial^{\mu} g_{\alpha 0 r \mu}(\omega, x) A^{r}(x)\right)\right] \\
& -\int_{0}^{\infty} d \omega \partial_{\mu} g^{\alpha l s \mu}(\omega, x) \partial_{l} X_{\alpha}(\omega, x) A_{s}(x) \\
& -\frac{1}{2} \int_{0}^{\infty} d \omega\left[\partial_{l} X_{\nu}(\omega, x) \partial^{l} X^{\nu}(\omega, x)-\omega^{2} X_{\nu}(\omega, x) X^{\nu}(\omega, x)\right],
\end{aligned}
$$

where the summation should be done over the indices $\mu, \nu, \alpha, \beta, s, r$ from 0 up to 3 and over $l$ from 1 up to 3. Using the commutation relation (88) and Hamiltonian of the total system $H\left(x_{0}\right)=\int d^{3} x h\left(x_{0}, \mathbf{x}\right)$, one can show, straightforwardly, the combination of the equation of motion of the conjugate pair $\Pi^{\nu}(x), A^{\nu}(x)$ in the Heisenberg picture leads to the relativistic covariant equation (75) for the four-vector potential of the electromagnetic field. As well, using the commutation relations (89) and (90) it can be shown that the combinations of the Heisenberg equations of the conjugate variable pairs $Q^{\alpha \beta}(\omega, x), Y^{\alpha \beta}(\omega, x)$ and $D^{\nu}(\omega, x), X^{\nu}(\omega, x)$ give us the relativistic covariant equations (76) and (77), respectively. Accordingly the relativistic covariant constitutive relations (82) and (83) of the anisotropic conductor polarizable and magnetizable medium are valid in quantum electrodynamics where now the antisymmetric electric-magnetic polarization tensor $K^{\mu \nu}(x)$, the free electric current density four-vector $J^{\nu}(x)$ of the medium and the strength tensor of the electromagnetic field are appeared as Hermitian operators. 


\section{Amooshahi}

The first part solutions $Y_{N}^{\alpha \beta}(\omega, x)$ and $X_{N}^{\nu}(\omega, x)$ in (78) and (79) can be expanded in terms of the plane waves as

$$
\begin{aligned}
Y_{N}^{\alpha \beta}(\omega, x)= & \sum_{\lambda_{1}, \lambda_{1}^{\prime}=0}^{3} \int_{-\infty}^{+\infty} d^{3} k \sqrt{\frac{\hbar}{2(2 \pi)^{3} \sqrt{k^{2}+\omega^{2}}}} \\
& \times\left[b_{\lambda_{1} \lambda_{1}^{\prime}}(\omega, \mathbf{k}) e^{i \mathbf{k} \cdot \mathbf{x}-i \sqrt{k^{2}+\omega^{2}} x_{0}}\right. \\
& \left.+b_{\lambda_{1} \lambda_{1}^{\prime}}^{\dagger}(\omega, \mathbf{k}) e^{-i \mathbf{k} \cdot \mathbf{x}+i \sqrt{k^{2}+\omega^{2}} x_{0}}\right] \eta^{\alpha \beta}\left(\lambda_{1}, \lambda_{1}^{\prime}, \mathbf{k}\right), \\
X_{N}^{\nu}(\omega, x)= & \sum_{\lambda=0}^{3} \int_{-\infty}^{+\infty} d^{3} k \sqrt{\frac{\hbar}{2(2 \pi)^{3} \sqrt{k^{2}+\omega^{2}}}} \\
& \times\left[a_{\lambda}(\omega, \mathbf{k}) e^{i \mathbf{k} \cdot \mathbf{x}-i \sqrt{k^{2}+\omega^{2}} x_{0}}\right. \\
& \left.+a_{\lambda}^{\dagger}(\omega, \mathbf{k}) e^{-i \mathbf{k} \cdot \mathbf{x}+i \sqrt{k^{2}+\omega^{2}} x_{0}}\right] e^{\nu}(\lambda, \mathbf{k}),
\end{aligned}
$$

where the four-vectors $e(\lambda, \mathbf{k}), \lambda=0,1,2,3$ for each wave vector $\mathbf{k}$ satisfy the completeness and the orthonormality relations (28) and (29). Also the antisymmetric tensors $\eta^{\alpha \beta}\left(\lambda_{1}, \lambda_{1}^{\prime}, \mathbf{k}\right), \lambda_{1}, \lambda_{1}^{\prime}=0,1,2,3$ for each wave vector $\mathbf{k}$ are defined in terms of the four vectors $e(\lambda, \mathbf{k})$ as in the relation (30) and obey the completeness and the orthonormality relations (31) and (32). In the expansions (93) and (94) the annihilation operators $b_{\lambda_{1} \lambda_{1}^{\prime}}(\omega, \mathbf{k}), a_{\lambda}(\omega, \mathbf{k})$ and the creation operators $b_{\lambda_{1} \lambda_{1}^{\prime}}^{\dagger}(\omega, \mathbf{k})$, $a_{\lambda}^{\dagger}(\omega, \mathbf{k})$ satisfy the commutation relations

$$
\begin{aligned}
& \quad\left[b_{\lambda_{1} \lambda_{1}^{\prime}}(\omega, \mathbf{k}), b_{\lambda_{2} \lambda_{2}^{\prime}}\left(\omega^{\prime}, \mathbf{k}^{\prime}\right)\right] \\
& \quad=\frac{1}{4}\left[g_{\lambda_{1} \lambda_{2}} g_{\lambda_{1}^{\prime} \lambda_{2}^{\prime}}-g_{\lambda_{1} \lambda_{2}^{\prime}} g_{\lambda_{1}^{\prime} \lambda_{2}}\right] \delta\left(\omega-\omega^{\prime}\right) \delta^{(3)}\left(\mathbf{k}-\mathbf{k}^{\prime}\right), \\
& {\left[a_{\lambda}(\omega, \mathbf{k}), a_{\lambda^{\prime}}^{\dagger}\left(\omega^{\prime}, \mathbf{k}^{\prime}\right)\right]=g_{\lambda \lambda^{\prime}} \delta^{(3)}\left(\mathbf{k}-\mathbf{k}^{\prime}\right) \delta\left(\omega-\omega^{\prime}\right)}
\end{aligned}
$$

which are compatible to the commutation relations (89) and (90), respectively. The expansions (93) and (94) can be used to express the total noise current density fourvector induced in the anisotropic conductor polarizable and magnetizable medium in terms of the ladder operators of the total system. Using the definitions of the noise electric-magnetic polarization tensor $K_{N}^{\mu \nu}(x)$ and the noise free electric current density four-vector $J_{N}^{\nu}(x)$ given by (12) and (47), the total noise current density four-vector induced in the medium in terms of the ladder operators of the total system clearly is

$$
\begin{aligned}
G_{N}^{\nu}(x) & =c \partial_{\mu} K_{N}^{\mu \nu}(x)+J_{N}^{\nu}(x) \\
& =\frac{c}{4 \pi} \int_{0}^{\infty} d \omega \sum_{\lambda_{1}, \lambda_{1}^{\prime}=0}^{3} \int_{-\infty}^{+\infty} d^{4} \kappa \sqrt{\frac{\hbar}{2(2 \pi)^{3} \sqrt{k^{2}+\omega^{2}}}} \delta\left(\kappa_{0}-\sqrt{k^{2}+\omega^{2}}\right)
\end{aligned}
$$




$$
\begin{aligned}
& \times\left[b_{\lambda_{1} \lambda_{1}^{\prime}}(\omega, \mathbf{k}) \partial_{\mu}\left(f^{\mu \nu \alpha \beta}(\omega, x) e^{-i \kappa_{r} x^{r}}\right)+\text { h.c. }\right] \eta_{\alpha \beta}\left(\lambda_{1}, \lambda_{1}^{\prime}, \mathbf{k}\right) \\
& +\frac{c}{4 \pi} \int_{0}^{\infty} d \omega \partial_{\mu} g^{\alpha \beta \nu \mu}(\omega, x) \sum_{\lambda=0}^{3} \int_{-\infty}^{+\infty} d^{4} \kappa \sqrt{\frac{\hbar}{2(2 \pi)^{3} \sqrt{k^{2}+\omega^{2}}}} \\
& \times \delta\left(\kappa^{0}-\sqrt{k^{2}+\omega^{2}}\right)\left[\left(-i \kappa_{\beta}\right) a_{\lambda}(\omega, \mathbf{k}) e^{-i \kappa_{r} x^{r}}+\text { h.c. }\right] e_{\alpha}(\lambda, \mathbf{k})
\end{aligned}
$$

where $\kappa=\left(\kappa_{0}, \mathbf{k}\right)$ is the wave four-vector in four-dimensional reciprocal space and $k=|\mathbf{k}|$. As we will see, the total noise current density four-vector will be appeared on the right-hand side of the quantum relativistic covariant wave equation of the four-vector potential of the electromagnetic field as a source term.

\subsection{The quantum relativistic covariant wave equation of the four-vector potential}

Using the wave equation (75) and the constitutive relations (82) and (83) the quantum relativistic covariant wave equation of the four-vector potential of the electromagnetic field in Minkowski space-time clearly can be written as

$$
\begin{array}{r}
-\partial_{\mu} \partial^{\mu} A^{\nu}(x)-8 \pi \partial_{\mu} \int_{-\infty}^{+\infty} d^{4} x^{\prime} \chi^{\mu \nu r s}\left(x, x^{\prime}\right) \partial_{r}^{\prime} A_{s}\left(x^{\prime}\right) \\
-\frac{8 \pi}{c} \int_{-\infty}^{+\infty} d^{4} x^{\prime} \sigma^{\nu r s}\left(x, x^{\prime}\right) \partial_{r}^{\prime} A_{s}\left(x^{\prime}\right)=\frac{4 \pi}{c} G_{N}^{\nu}(x),
\end{array}
$$

where $G_{N}^{\nu}(x)$ is the total noise current density four-vector induced in the anisotropic conductor polarizable and magnetizable medium and is defined by (96). In (97) the tensors $\chi^{\mu \nu r s}\left(x, x^{\prime}\right)$ and $\sigma^{\nu r s}\left(x, x^{\prime}\right)$ are the susceptibility and the conductivity tensors of the anisotropic conductor polarizable and magnetizable medium, respectively. In writing the wave equation (97) the antisymmetry relations

$$
\chi^{\mu \nu r s}\left(x, x^{\prime}\right)=-\chi^{\mu \nu s r}\left(x, x^{\prime}\right), \quad \sigma^{\nu r s}\left(x, x^{\prime}\right)=-\sigma^{\nu s r}\left(x, x^{\prime}\right)
$$

have been used. The quantum relativistic wave equation (97) can be solved for a homogeneous anisotropic conductor magneto-dielectric medium. In this case the coupling tensors $f^{\mu \nu \alpha \beta}(\omega, x)$ and $\partial_{\mu} g^{\alpha \beta s \mu}(\omega, x)$ applied in the Lagrangian density of the total system $(74)$ are independent of the space-time. That is, we can write for the homogeneous anisotropic bulk medium $f^{\mu \nu \alpha \beta}(\omega, x) \equiv f^{\mu \nu \alpha \beta}(\omega)$ and $\partial_{\mu} g^{\alpha \beta s \mu}(\omega, x) \equiv g^{\alpha \beta s}(\omega)$. Therefore, in this case according to the definitions (13) and (48) the susceptibility and the conductivity tensors of the medium are dependent only on the difference $x-x^{\prime}$ in four-dimensional Minkowski space-time and the wave equation (97) can be rewritten as

$$
\begin{gathered}
-\partial_{\mu} \partial^{\mu} A^{\nu}(x)-8 \pi \partial_{\mu} \int_{-\infty}^{+\infty} d^{4} x^{\prime} \chi^{\mu \nu r s}\left(x-x^{\prime}\right) \partial_{r}^{\prime} A_{s}\left(x^{\prime}\right) \\
-\frac{8 \pi}{c} \int_{-\infty}^{+\infty} d^{4} x^{\prime} \sigma^{\nu r s}\left(x-x^{\prime}\right) \partial_{r}^{\prime} A_{s}\left(x^{\prime}\right)=\frac{4 \pi}{c} G_{N}^{\nu}(x) .
\end{gathered}
$$




\section{Amooshahi}

This equation can be solved easily using the four-dimensional Fourier transform. Let us write the four-vector potential $A^{\nu}(x)$ and $G_{N}^{\nu}(x)$ in terms of their fourdimensional Fourier transform as

$$
\begin{aligned}
A^{\nu}(x) & =\frac{1}{(2 \pi)^{4}} \int_{-\infty}^{+\infty} d^{4} \kappa \underline{A}^{\nu}(\kappa) e^{-i \kappa_{\mu} x^{\mu}}, \\
G_{N}^{\nu}(x) & =\frac{1}{(2 \pi)^{4}} \int_{-\infty}^{+\infty} d^{4} \kappa \underline{G}_{N}^{\nu}(\kappa) e^{-i \kappa_{\mu} x^{\mu}} .
\end{aligned}
$$

Then the wave equation (99) can be written as

$$
\begin{gathered}
\underline{\Lambda}^{\nu \beta}(\kappa) \underline{A}_{\beta}(\kappa)=\frac{4 \pi}{c} \underline{G}_{N}^{\nu}(\kappa), \\
\underline{\Lambda}^{\nu \beta}(\kappa)=\left[\left(\kappa_{\mu} \kappa^{\mu}\right) g^{\nu \beta}+8 \pi\left(\kappa_{\mu} \kappa_{r}\right) \underline{\chi}^{\mu \nu r \beta}(\kappa)-\frac{8 \pi}{c}\left(i \kappa_{r}\right) \underline{\sigma}^{\nu r \beta}(\kappa)\right]
\end{gathered}
$$

in four-dimensional reciprocal space where $g^{\nu \beta}$ is the Minkowski space-time metric and $\chi^{\mu \nu r \beta}(\kappa)$ and $\underline{\sigma}^{\nu r \beta}(\kappa)$ are the four-dimensional Fourier transforms of the susceptibility and the conductivity tensors of the medium defined by

$$
\begin{aligned}
\chi^{\mu \nu r \beta}(x) & =\frac{1}{(2 \pi)^{4}} \int_{-\infty}^{+\infty} d^{4} \kappa \underline{\chi}^{\mu \nu r \beta}(\kappa) e^{-i \kappa_{\mu} x^{\mu}}, \\
\sigma^{\nu r \beta}(x) & =\frac{1}{(2 \pi)^{4}} \int_{-\infty}^{+\infty} d^{4} \kappa \underline{\sigma}^{\nu r \beta}(\kappa) e^{-i \kappa_{\mu} x^{\mu}} .
\end{aligned}
$$

Using the expression (96) for the total noise electric current density four-vector and according to (101) the space-time dependent of the quantum four-vector potential of the electromagnetic field can be easily obtained in terms of the ladder operators of the total system as

$$
\begin{aligned}
A^{\nu}(x)= & \frac{1}{(2 \pi)^{4}} \int_{-\infty}^{+\infty} d^{4} \kappa \underline{A}^{\nu}(\kappa) e^{-i \kappa_{\mu} x^{\mu}} \\
= & \frac{4 \pi}{c} \frac{1}{(2 \pi)^{4}} \int_{-\infty}^{+\infty} d^{4} \kappa\left[\left[\underline{\Lambda}^{-1}(\kappa)\right]_{\beta}^{\nu} \underline{G}_{N}^{\beta}(\kappa)\right] e^{-i \kappa_{\mu} x^{\mu}} \\
= & \int_{0}^{\infty} d \omega f^{\mu \beta r s}(\omega) \int_{-\infty}^{+\infty} d^{4} \kappa \sqrt{\frac{\hbar}{2(2 \pi)^{3} \sqrt{k^{2}+\omega^{2}}}} \delta\left(\kappa_{0}-\sqrt{k^{2}+\omega^{2}}\right) \\
& \times \sum_{\lambda_{1}, \lambda_{1}^{\prime}=0}^{3}\left[\left(\left[\Lambda^{-1}(\kappa)\right]_{\beta}^{\nu} b_{\lambda_{1} \lambda_{1}^{\prime}}(\omega, \mathbf{k})\left(-i \kappa_{\mu}\right) e^{-i \kappa_{\alpha} x^{\alpha}}+\text { h.c. }\right) \eta_{r s}\left(\lambda_{1}, \lambda_{1}^{\prime}, \mathbf{k}\right)\right] \\
& +\int_{0}^{\infty} d \omega g^{r \mu \beta}(\omega) \int_{-\infty}^{+\infty} d^{4} \kappa \sqrt{\frac{\hbar}{2(2 \pi)^{3} \sqrt{k^{2}+\omega^{2}}}} \delta\left(\kappa_{0}-\sqrt{k^{2}+\omega^{2}}\right) \\
& \times \sum_{\lambda=0}^{3}\left[\left(\left[\Lambda^{-1}(\kappa)\right]_{\beta}^{\nu} a_{\lambda}(\omega, \mathbf{k})\left(-i \kappa_{\mu}\right) e^{-i \kappa_{s} x^{s}}+\text { h.c. }\right) e_{r}(\lambda, \mathbf{k})\right],
\end{aligned}
$$

where the summation should be done over the indices $\mu, \beta, r, s$ from 0 up to 3 . 


\section{Conclusion}

Modeling an anisotropic polarizable and magnetizable medium by a continuum collection of antisymmetric tensor fields of the second rank in Minkowski space-time, a canonical relativistic quantization of the electromagnetic field in the presence of such a medium was introduced. The choice of the antisymmetric tensor fields to describe the polarization and the magnetization properties of the medium was in correspondence to the nonrelativistic canonical quantization of the electromagnetic field in the presence of a bi-anisotropic magneto-dielectric medium. A Lagrangian density, that was invariant under Poincaré transformation, was proposed for the total system (the electromagnetic field plus the anisotropic magneto-dielectric). The electromagnetic field was coupled to the anisotropic magneto-dielectric medium by a continuum set of the tensor fields of the fourth rank in Minkowski spacetime. Some antisymmetry conditions were imposed on the coupling tensors of the electromagnetic field and the magneto-dielectric medium. The antisymmetry features of the coupling tensor fields were necessary in order to consist of the canonical relativistic quantization scheme in viewpoints of physical and mathematical concepts. The classical relativistic covariant Euler-Lagrange equation of the fourvector potential of the electromagnetic field gave the correct wave equation for the four-vector potential in the presence of a magneto-dielectric medium in Lorentz gauge. The antisymmetric electric-magnetic polarization tensor of the anisotropic magneto-dielectric medium was defined in terms of the antisymmetric tensor fields modeling the medium and the coupling tensors that couple the electromagnetic field to the anisotropic magneto-dielectric medium. The relativistic covariant constitutive relation of the anisotropic polarizable and magnetizable medium, relating the antisymmetric electric-magnetic polarization tensor fields of the medium to the strength tensor of the electromagnetic field, was extracted. The susceptibility tensor of the anisotropic magneto-dielectric medium was obtained in terms of the retarded Green function and the coupling tensors that coupled the electromagnetic field to the medium. The susceptibility tensor of the medium was a tensor field of the fourth rank, in Minkowski space-time, and was antisymmetric with respect to the first pair and the last pair of its indices. Such a tensor, in Minkowski space-time, has 36 independent components which is just the needed component numbers to relate each Cartesian component of the electric polarization and the magnetic polarization densities of the medium, separately, to both the electric and the magnetic fields. It was shown that the susceptibility tensor for a homogeneous anisotropic bulk magneto-dielectric medium satisfies the Kramers-Kronig relations in frequency domain. A canonical relativistic quantization of both the electromagnetic field and the anisotropic magneto-dielectric medium was provided, in a standard way, in the Gupta-Bleuler formalism. The relativistic covariant constitutive relation of the anisotropic magneto-dielectric medium was remained valid in relativistic quantum electrodynamics. The noise antisymmetric electric-magnetic polarization tensor field of the magneto-dielectric medium was appeared in the constitutive relation 
of the medium. The noise fields are necessary for a consistent quantization of the classical fields in the presence of absorbing media. The noise antisymmetric electricmagnetic polarization tensor field of the medium was expressed in terms of the ladder operators of the total system and the coupling tensor fields that coupled the electromagnetic field to the anisotropic magneto-dielectric medium.

The canonical relativistic quantization method of the electromagnetic field was generalized in the presence of an anisotropic conductor medium that is not polarizable or magnetizable. In this case the conductor medium was modeled by a continuum collection of vector fields in Minkowski space-time. A Lagrangian density was provided for the total system (the electromagnetic field plus the conductor medium) which was invariant under Poincaré transformations. The anisotropic conductor medium was coupled to the electromagnetic field by a continuum set of the tensor fields of the fourth rank in Minkowski space-time. Some antisymmetry conditions were imposed on the coupling tensor fields. The antisymmetry properties of the coupling tensors were sufficient conditions for the conservation of the free electric charges induced in the conductor medium. The classical relativistic covariant Euler-Lagrange equation of the four-vector potential of electromagnetic field was coincided the correct equation of the four-vector potential in the presence of a conductor medium in Lorentz gauge. The free electric current density four-vector induced in the conductor medium was defined in terms of the vector fields modeling the medium and the coupling tensors that coupled the conductor medium to the electromagnetic field. The relativistic covariant constitutive relation of the anisotropic conductor medium, relating the free electric current density four-vector induced in the conductor medium to the strength tensor of the electromagnetic field, was obtained. The conductivity tensor of the anisotropic conductor medium was obtained in terms of the spatial-temporal derivatives of the retarded Green function and the coupling tensors that coupled the electromagnetic field to the anisotropic conductor medium. The conductivity tensor of the medium was a tensor field of the third rank, in Minkowski space-time, which was antisymmetric with respect to the last two indices. Such a tensor, in Minkowski space-time, has 24 independent components which is precisely the needed component numbers to relate each component of the electric current density four-vector, induced in the conductor medium, to both the electric and the magnetic fields. For a homogeneous anisotropic bulk conductor medium, the conductivity tensor of the medium was decomposed into two terms. The first term was contained the spatial derivatives of the retarded Green function and the second term was contained the temporal derivative of the retarded Green function. The real and imaginary parts of the first term obeyed the Kramers-Kronig relations in real frequency domain. The real and imaginary parts of the second term, in real frequency domain, were related to each other by two relations other than the Kramers-Kronig relations. This was so because the second term was not an analytical complex function on the real axis of the complex plan of frequencies. A canonical relativistic quantization of both the electromagnetic field and the anisotropic conductor medium was 
achieved in the Gupta-Bleuler framework. The relativistic covariant constitutive relation of the conductor medium was remained valid in the relativistic quantum electrodynamics. The noise free electric current density four-vector, induced in the conductor medium, was found in the constitutive relation of the medium. The noise free electric current density four-vector was written in terms of the ladder operators of the total system and the coupling tensors that couple the electromagnetic field to anisotropic conductor medium.

The canonical relativistic quantization scheme of the electromagnetic field was applied, straightforwardly, in the presence of an anisotropic conductor polarizable and magnetizable medium. The anisotropic conductor magneto-dielectric medium was modeled by a continuum collection of vector fields and a continuum collection of antisymmetric tensor fields of the second rank in Minkowski space-time. The collection of the vector fields describe the conductivity property of the medium and the collection of the antisymmetric tensor fields describe the polarization and the magnetization properties of the medium. A Lagrangian density was proposed for the total system (the electromagnetic field plus the anisotropic conductor magnetodielectric medium) that was invariant under Poincaré transformation. Two different coupling tensor field sets of the fourth rank, in Minkowski space-time, were applied in the interaction part of the Lagrangian density of the total system. One set coupled the electromagnetic field to the vector fields describing the conductivity property of the medium. Another set coupled the electromagnetic field to the antisymmetric tensor fields describing the polarization and the magnetization properties of the medium. Some antisymmetry conditions were imposed on the coupling tensor fields of the electromagnetic field and the anisotropic conductor magnetodielectric medium. The antisymmetry features of the coupling tensors gave the conservation law of the total electric charges induced in the anisotropic conductor magneto-dielectric medium. The classical relativistic covariant Euler-Lagrange equation of the four-vector potential of the electromagnetic field was coincided the correct equation of the four-vector potential in the presence of an anisotropic conductor magneto-dielectric medium in Lorentz gauge. The free electric current density four-vector, induced in the conductor magneto-dielectric medium, was defined in terms of the vector fields describing the conductivity of the medium and the coupling tensors that coupled the electromagnetic field to the vector fields. The antisymmetric electric-magnetic polarization tensor of the anisotropic conductor magneto-dielectric medium was written in terms of the antisymmetric tensor fields describing the polarization and the magnetization properties of the medium and the coupling tensors that coupled the electromagnetic field to the antisymmetric tensor fields. Two relativistic covariant constitutive relations for the anisotropic conductor polarizable and magnetizable medium were obtained. One of the constitutive relations related the free electric current density four-vector, induced in the medium, to the strength tensor of the electromagnetic field. Another constitutive relation related the antisymmetric electric-magnetic polarization tensor of 
the medium to the strength tensor of the electromagnetic field. The susceptibility tensor of the anisotropic conductor magneto-dielectric medium was expressed in terms of the retarded Green function and the coupling tensors which coupled the electromagnetic field to the antisymmetric tensor fields describing the polarization and the magnetization properties of the medium. The susceptibility tensor of the medium possessed the same mathematical and physical features of the susceptibility tensor of the anisotropic magneto-dielectric (but not conductor) medium. The conductivity tensor of the anisotropic conductor magneto-dielectric medium was obtained in terms of the spatial-temporal derivatives of the retarded Green function and the coupling tensors that coupled the electromagnetic field to the vector fields describing the conductivity property of the medium. The conductivity tensor of the medium possessed the same physical and mathematical features of the anisotropic conductor (but not polarizable and magnetizable) medium mentioned above. Using the Lagrangian density of the total system (the electromagnetic field plus the anisotropic conductor magneto-dielectric medium) a canonical relativistic quantization of both the electromagnetic field and the medium was outlined in the Gupta-Bleuler formalism. The two relativistic covariant constitutive relations of the anisotropic conductor polarizable and magnetizable medium were remained valid in relativistic quantum electrodynamics. The total noise electric current density four-vector, induced in the medium, was expressed in terms of the ladder operators of the total system and the coupling tensors that coupled the electromagnetic field to the medium. The quantum relativistic covariant wave equation of the four-vector potential of the electromagnetic field was obtained in the presence of the anisotropic conductor polarizable and magnetizable medium. The total noise electric current density four-vector, induced in the anisotropic conductor magnetodielectric medium, was appeared on the right-hand side of the quantum relativistic covariant wave equation of the four-vector potential as a source term. The quantum relativistic wave equation of the four-vector potential was solved in the presence of a homogeneous anisotropic conductor polarizable and magnetizable medium using the four-dimensional Fourier transform technique.

\section{References}

1. R. Matloob, R. Loudon, S. M. Barnett and J. Jeffers, Phys. Rev. A 52, 4823 (1995).

2. T. Gruner and D. G. Welsch, Phys. Rev. A 53, 1818 (1996).

3. R. Matloob and R. Loudon, Phys. Rev. A 53, 4567 (1996).

4. H. T. Dung, L. Knöll and D. G. Welsch, Phys. Rev. A 57, 3931 (1998).

5. S. Scheel, L. Knöll and D. G. Welsch, Phys. Rev. A 58, 700 (1998).

6. R. Matloob, Phys. Rev. A 60, 50 (1999).

7. S. Scheel and D. G. Welsch, arXiv:quant-ph/9906001v2.

8. L. Knöll, S. Scheel and D. G. Welsch, arXiv:quant-ph/0006121v5.

9. C. Raabe, S. Scheel and D. G. Welsch, Phys. Rev. A 75, 053813 (2007).

10. H. T. Dung, S. Y. Buhmann, L. Knöll and D. G. Welsch, Phys. Rev. A 68, 043816 (2003). 
11. R. Matloob, R. Loudon, M. Artoni, S. M. Barnett and J. Jeffers, Phys. Rev. A 55, 1623 (1997).

12. C. Raabe and D. G. Welsch, arXiv:0710.2867v1 [quant-ph].

13. M. S. Tomas and Z. Lenac, Phys. Rev. A 56, 4197 (1997).

14. S. Scheel, L. Knöll, D. G. Welsch and S. M. Barnett, Phys. Rev. A 60, 1590 (1999).

15. S. Scheel, L. Knöll and D. G. Welsch, Phys. Rev. A 60, 4094 (1999).

16. M. S. Tomas and Z. Lenak, Phys. Rev. A 60, 2431 (1999).

17. H. T. Dung, L. Knöll and D. G. Welsch, Phys. Rev. A 62, 053804 (2000).

18. H. T. Dung, L. Knöll and D. G. Welsch, Phys. Rev. A 64, 013804 (2001).

19. M. S. Tomas, Phys. Rev. A 63, 053811 (2001).

20. R. Matloob, Phys. Rev. A 72, 062103 (2005).

21. H. T. Dung, S. Y. Buhmann and D. G. Welsch, Phys. Rev. A 74, 023803 (2006).

22. C. Raabe and D. G. Welsch, arXiv:0710.2867v1 [quant-ph].

23. M. Khanbekyan, D. G. Welsch, C. DiFidio and W. Vogel, Phys. Rev. A 78, 013822 (2008).

24. C. DiFidio, W. Vogel, M. Khanbekyan and D. G. Welsch, Phys. Rev. A 77, 043822 (2008).

25. R. Matloob, A. Keshavarz and D. Sedighi, Phys. Rev. A 60, 3410 (1999).

26. R. Matloob, Phys. Rev. A 60, 3421 (1999).

27. R. Matloob and H. Falinejad, Phys. Rev. A 64, 042102 (2001).

28. M. S. Tomas, Phys. Rev. A 66, 052103 (2002).

29. C. Raabe, L. Knöll and D. G. Welsch, Phys. Rev. A 68, 033810 (2003).

30. C. Raabe, L. Knöll and D. G. Welsch, Phys. Rev. A 68, 049902 (2003).

31. C. Raabe, L. Knöll and D. G. Welsch, arXiv:quant-ph/0309179v1.

32. S. Y. Buhmann, L. Knöll, D. G. Welsch and H. T. Dung, Phys. Rev. A 70, 052117 (2004).

33. R. Matloob, Phys. Rev. A 70, 062110 (2004).

34. C. Raabe, L. Knöll and D. G. Welsch, arXiv:quant-ph/0212154v2.

35. C. Raabe and D. G. Welsch, Phys. Rev. A 71, 013814 (2005).

36. M. S. Tomas, Phys. Rev. A 71, 0601101 (2005).

37. M. S. Tomas, Phys. Rev. A 72, 034104 (2005).

38. C. Raabe, S. Scheel and D. G. Welsch, Phys. Rev. A 71, 013814 (2005).

39. C. Raabe, S. Scheel and D. G. Welsch, Phys. Rev. A 73, 063822 (2006).

40. C. Raabe and D. G. Welsch, Phys. Rev. A 73, 063822 (2006).

41. C. Raabe and D. G. Welsch, arXiv:quant-ph/0602059v1.

42. C. Raabe and D. G. Welsch, Hyperfine Interact. 172, 142 (2006).

43. Z. Lenac and M. S. Tomas, Phys. Rev. A 75, 042101 (2007).

44. S. Y. Buhmann and D. G. Welsch, arXiv:quant-ph/0608118v2.

45. S. Y. Buhmann, L. Knöll, D. G. Welsch and H. T. Dung, arXiv:quant-ph/0403128v3.

46. Z. Lenac and M. S. Tomas, Phys. Rev. A 78, 023834 (2008).

47. S. Y. Buhmann and D. G. Welsch, Phys. Rev. A 77, 012110 (2008).

48. A. Samabale, S. Y. Buhmann, H. T. Dung and D. G. Welsch, Phys. Rev. A 80, 051801 (2009).

49. S. Scheel and S. Y. Buhmann, Phys. Rev. A 80, 042902 (2009).

50. A. Sambale, S. Y. Buhmann and S. Scheel, Phys. Rev. A 81, 012509 (2010).

51. S. A. Ellingsen, S. Y. Buhmann and S. Scheel, Phys. Rev. A 84, 060501 (2011).

52. R. Matloob and A. Ghaffari, Phys. Rev. A 70, 052116 (2004).

53. S. I. Maslovski and M. G. Silveirinha, Phys. Rev. A 84, 062509 (2011).

54. M. Amooshahi, J. Math. Phys. 50, 062301 (2009).

55. J. D. Jackson, Classical Electrodynamics (John Wiley, 1998). 
56. M. Amooshahi, Eur. Phys. J. D 69, 66 (2015).

57. M. Amooshahi, Int. J. Theor. Phys. 55, 3761 (2016).

58. G. S. He and S. H. Liu, Physics of Nonlinear Optics (World Scientific, 1999).

59. M. Amooshahi, Eur. Phys. J. D 54, 115 (2009).

60. W. Greiner and J. Reinhardt, Field Quantization (Springer-Verlag, Berlin, 1996). 